\title{
ZIKV disrupts placental ultrastructure and drug transporter expression in mice
}

3 Cherley Borba Vieira de Andrade $^{1}$, Victoria Regina de Siqueira Monteiro ${ }^{1}$, Sharton

4 Vinicius Antunes Coelho ${ }^{2}$, Hanailly Ribeiro Gomes ${ }^{1}$, Ronny Paiva Campos de Sousa ${ }^{1}$,

5 Veronica Muller de Oliveira Nascimento ${ }^{1}$, Flavia Fonseca Bloise ${ }^{1}$, Stephen

6 Matthews $^{3,4,5,6}$, Enrrico Bloise ${ }^{7}$, Luciana Barros de Arruda $^{2}$, Tania Maria Ortiga-

7 Carvalho ${ }^{1 *}$.

$9{ }^{1}$ Institute of Biophysics Carlos Chagas Filho, Federal University of Rio de Janeiro, Rio

10 de Janeiro, RJ, Brazil.

$11{ }^{2}$ Institute of Microbiology Paulo de Góes, Federal University of Rio de Janeiro, Rio de

12 Janeiro, RJ, Brazil.

$13{ }^{3}$ Department of Physiology, Faculty of Medicine, University of Toronto, Toronto,

14 Canada.

$15{ }^{4}$ Department of Obstetrics \& Gynecology, Faculty of Medicine, University of Toronto,

16 Toronto, Canada.

$17{ }^{5}$ Department of Medicine, Faculty of Medicine, University of Toronto, Toronto,

18 Canada.

$19{ }^{6}$ Lunenfeld-Tanenbaum Research Institute, Mount Sinai Hospital, Toronto, Canada.

$20{ }^{7}$ Department of Morphology, Federal University of Minas Gerais, Belo Horizonte, BH,

21 Brazil.

$22 *$ Correspondence:

23 Tania Maria Ortiga-Carvalho (TMO-C)

24 taniaort@biof.ufrj.br

26 Key words: ZIKV, placenta, P-glycoprotein (P-gp), breast cancer resistance

27 protein (Bcrp), Abca1, Abcg1, ultrastructure, cytokine, chemokine 


\section{Abstract}

30 Congenital Zika virus (ZIKV) infection can induce fetal brain abnormalities. Here, we

31 investigated whether maternal ZIKV infection affects placental physiology and

32 metabolic transport potential and impacts the fetal outcome, regardless of viral presence

33 in the fetus at term. Low $\left(10^{3}\right.$ PFU-ZIKVPE243; low ZIKV) and high $\left(5 \times 10^{7}\right.$ PFU-

34 ZIKVPE243; high ZIKV) virus titers were injected into immunocompetent (ICompetent

35 C57BL/6) and immunocompromised (ICompromised A129) mice at gestational day

36 (GD) 12.5 for tissue collection at GD18.5 (term). High ZIKV elicited fetal death rates of

$3766 \%$ and $100 \%$, whereas low ZIKV induced fetal death rates of $0 \%$ and $60 \%$ in

38 C57BL/6 and A129 dams, respectively. All surviving fetuses exhibited intrauterine

39 growth restriction (IUGR) and decreased placental efficiency. High-ZIKV infection in

40 C57BL/6 and A129 mice resulted in virus detection in maternal spleens and placenta,

41 but only A129 fetuses presented virus RNA in the brain. Nevertheless, pregnancies in

42 both strains produced fetuses with decreased head sizes $(\mathrm{p}<0.05)$. Low-ZIKV-A129

43 dams had higher IL-6 and CXCL1 levels ( $\mathrm{p}<0.05)$, and their placentas showed increased

44 CCL-2 and CXCL-1 contents ( $\mathrm{p}<0.05)$. In contrast, low-ZIKV-C57BL/6 dams had an

45 elevated CCL2 serum level and increased type I and II IFN expression in the placenta.

46 Notably, less abundant microvilli and mitochondrial degeneration were evidenced in the

47 placental labyrinth zone (Lz) of ICompromised and high-ZIKV-ICompetent mice but

48 not in low-ZIKV-C57BL/6 mice. In addition, decreased placental expression of the drug

49 transporters P-glycoprotein (P-gp) and breast cancer resistance protein (Bcrp) and the

50 lipid transporter Abca1 was detected in all ZIKV-infected groups, but Bcrp and Abca1

51 were only reduced in ICompromised and high-ZIKV ICompetent mice. Our data

52 indicate that gestational ZIKV infection triggers specific proinflammatory responses

53 and affects placental turnover and transporter expression in a manner dependent on 
54 virus concentration and maternal immune status. Placental damage may impair proper

55 fetal-maternal exchange function and fetal growth/survival, likely contributing to 56 congenital Zika syndrome.

\section{Introduction}

Congenital Zika virus (ZIKV) infection can be associated with adverse pregnancy outcomes. Neonates born from ZIKV-positive pregnancies may develop severe neurological abnormalities, placental pathologies and intrauterine growth restriction (IUGR), among other complications (1). ZIKV vertical transmission has become a major public health issue worldwide, especially in Brazil, where more than 200,000 ZIKV-positive cases have been confirmed and over 2,000 congenital microcephaly births have been reported (2-6). These numbers represent a 20 -fold rise in the incidence of congenital microcephaly in Brazil during the years of the ZIKV pandemic, with similar increases reported elsewhere in Latin America $(2,3,7)$. Importantly, while the ZIKV pandemic is currently thought to be controlled, evidence points to a possible silent ZIKV spread across the Americas $(8,9)$, highlighting the need for improved knowledge of the possible routes of vertical ZIKV transmission and its association with disruptive inflammatory and developmental phenotypes and the need for new avenues of prevention and treatment.

Previous studies have investigated the possible pathways involved in vertical ZIKV transmission. Miranda and colleagues (10) showed that in humans, ZIKV infection changed the pattern of tight junction proteins, such as claudin-4, in syncytiotrophoblasts. Jurado et al. (2016) suggested that the migratory activities of Hofbauer cells (feto-placental macrophages) could help disseminate ZIKV to the fetal brain (11). Other recent studies have shown that placental villous fibroblasts, cytotrophoblasts, endothelial cells and Hofbauer cells are permissive to ZIKV, and 
79 placentae from ZIKV-infected women had chorionic villi with a high mean diameter

80 (11-14). Furthermore, in 2019, Rathore et al. demonstrated that pregnant mice carrying

81 high levels of antibodies against dengue virus (DENV) exhibited increased ZIKV

82 vertical transmission associated with severe microcephaly-like syndrome,

83 demonstrating another possible mechanism of antibody-dependent vertical ZIKV

84 transmission (15). However, at present, further studies are required to identify the

85 precise mechanism of maternal-fetal ZIKV transmission.

86 Many mouse models have been developed to identify how ZIKV overcomes

87 placental defenses. Initially, limited information was obtained due to the apparent

88 inability of the virus to infect wild-type (WT) mice (16). ZIKV NS5 targets the

89 interferon signaling pathway in humans but not in mice (17). Thus, WT mice show no

90 clear evidence of clinical disease $(17,18)$ and are of limited use in modeling the disease.

91 However, mice lacking an interferon signaling response show evidence of disease and

92 have been widely used to investigate ZIKV infection during pregnancy $(8,17,19)$.

93 The interferon system, especially type III interferon, is a key mechanism of host

94 defense and a viral target for immune evasion (20). Type III interferons have a role in

95 protection against ZIKV infection in human syncytiotrophoblasts from term placenta

96 (21). Luo et al. have shown that inhibition of Toll-like receptors 3 and 8 inhibits the

97 cytokine output of ZIKV-infected trophoblasts (22). In addition, viral replication

98 coincides with the induction of proinflammatory cytokines, such as interleukin [IL]-6.

99 This cytokine has a crucial role in inflammation and affects the homeostatic processes

100 related to tissue injury and activation of stress-related responses $(23,24)$. ZIKV infection

101 can trigger an inflammatory response with IL-6 release $(11,25)$.

102 Maternal infection has profound effects on placental permeability to drugs and 103 environmental toxins. Changes in the expression and function of specific $\mathrm{ABC}$ 
104 transporters in the placenta and yolk sac following infective and inflammatory stimuli

105 have been demonstrated (26-30). ABC transporters are efflux transporters that control

106 the biodistribution of several endogenous and exogenous substrates, including

107 xenobiotics (antiretrovirals and synthetic glucocorticoids), steroid hormones (estrogens

108 and androgens), nutrients (folate and cholesterol) and immunological factors

109 (chemokines and cytokines) within the maternal-fetal interface (31). The best described

$110 \mathrm{ABC}$ transporters in the placenta are P-glycoprotein (P-gp; also known as multidrug

111 resistance protein 1, MDR1), breast cancer resistance protein (Bcrp) and the lipid Abca1

112 and Abcg1 transporters. P-gp and Bcrp transporters are responsible for preventing fetal

113 accumulation of xenobiotics and environmental toxins that may be present in the

114 maternal circulation, whereas Abca1 and Abcg1 control the placental exchange of

115 cytotoxic oxysterol and lipid permeability throughout pregnancy; therefore, they play an

116 important role in fetal protection and placental lipid homeostasis (26).

117 Despite the limited number of studies showing ZIKV infection in

118 immunocompetent mice, intrauterine inoculation with a high virus titer was previously

119 demonstrated to result in decreased fetal viability, with worse outcomes following

120 infection in early gestation (32). In another report, intravenous infection on a very early

121 embryonic day resulted in fetal demise even though the virus was not found in the fetal

122 compartment in most of the treated animals (33). In the present study, we hypothesize

123 that maternal exposure to ZIKV affects placental function, including placental

124 ultrastructure and $\mathrm{ABC}$ transporter (P-gp, Bcrp, Abca1 and Abcg1) protein expression,

125 even in the absence of vertical transmission and that these effects are dependent on viral

126 infective titers and maternal immune status. 


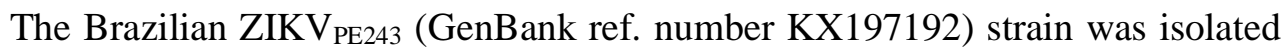

130 from a febrile case during the ZIKV outbreak in the state of Pernambuco, Brazil and

131 was kindly provided by Dr Ernesto T. Marques Jr. (Centro de Pesquisa Aggeu

132 Magalhães, FIOCRUZ, PE). Viruses were propagated in C6/36 cells, and viral titers

133 were determined by plaque assays in Vero cells, as previously described (34).

134 Supernatants of noninfected C6/36 cells cultured under the same conditions were used

135 as mock controls.

\section{$136 \quad 2.2$ Animal experimentation and study design}

137 Two mouse strains were used in the study: immunocompetent (ICompetent)

138 C57BL/6 and immunocompromised (ICompromised) (type 1 Ifnr-deficient) A129

139 strains. Since we were unable to consistently produce viable pregnancies by mating

140 A129 males and females in our experimental settings, we mated A129 females $(n=15)$

141 with C57BL/6 males $(\mathrm{n}=4)$ to produce ICompromised C57BL6/A129 pregnancies,

142 whereas ICompetent C57BL/6 pregnancies were obtained by mating male $(n=6)$ and

143 female ( $\mathrm{n}=35)$ C57BL/6 mice (8-10 weeks old). Animals were kept in a controlled

144 temperature room $\left(23^{\circ} \mathrm{C}\right)$ with a light/dark cycle of 12 hours and ad libitum access to

145 water and food. After detection of the proestrous/estrous phase via vaginal cytology,

146 copulation was confirmed by visualization of the vaginal plug and considered

147 gestational day 0.5 (GD0.5). Maternal weight was monitored for confirmation of

148 pregnancy; thus, females were weighed on GD0.5 and GD12.5, and females with a

149 weight gain greater than $3 \mathrm{~g}$ were considered pregnant and entered randomly in the

150 experimental groups. Experimental protocols were approved by the Animal Care

151 Committee of the Health Sciences Center, Federal University of Rio de Janeiro (CEUA-

152 036/16 and 104/16) and registered with the Brazilian National Council for Animal

153 Experimentation Control. 
On GD12.5, pregnant mice (ICompetent and ICompromised pregnancies) were

155 injected with a single intravenous (i.v.) titer of ZIKV or mock control. ICompetent and

156 ICompromised pregnant mice were randomly subdivided into three experimental

157 groups: the mock (control) group, which received an injection of supernatant from

158 noninfected C6/36 cells (ICompetent mock and ICompromised mock); the high-ZIKV-

159 titer group, inoculated with $5 \times 10^{7}$ plaque-forming units (PFU) of ZIKV

160 (ICompetent high and ICompromised high); and the ZIKV low-titer group, injected 161 with $10^{3} \mathrm{PFU}$ of $\mathrm{ZIKV}_{\mathrm{PE} 243}$ (ICompetent low and ICompromised low).

162 On the morning of GD18.5, all animals were euthanized with a sodium 163 phenobarbital overdose of $300 \mathrm{mg} / \mathrm{kg}$. Maternal blood was collected via cardiac 164 puncture, centrifuged (10 $\mathrm{min}, 4000 \mathrm{~g})$ and stored at $-20^{\circ} \mathrm{C}$. The maternal brain and 165 spleen and all placentae and all fetuses were dissected, collected and weighed, followed 166 by fetal head isolation and measurement. The three placentae closest to the mean weight 167 in a litter were selected for further analysis and cut in half using umbilical cord insertion 168 as a reference (35-37). One-half of the placental disk was frozen in liquid nitrogen for 169 qPCR, and the other half was fixed overnight in buffered paraformaldehyde (4\%, 170 Sigma-Aldrich, Brazil) for ultrastructural and protein expression/localization analysis.

171 Matched fetal heads, maternal brains and spleens were frozen in liquid nitrogen for 172 qPCR. Of important note, all fetuses obtained from ICompromised pregnancies were 173 heterozygous.

\section{$174 \quad 2.3$ ZIKV RNA quantification via RT-qPCR}

ZIKV load was evaluated in maternal blood, brains and spleens and in the

176 placentae and fetal heads. Brains, spleens, placentae and fetal heads were macerated in 177 RPMI medium (Gibco ${ }^{\mathrm{TM}}$ RPMI 1640 Medium) normalized by the ratio of $0.2 \mathrm{mg}$ of 178 tissue to every $1 \mu 1$ of medium and plotted per gram of tissue. The macerated volume 
179 was centrifuged at $4500 \mathrm{~g}$ for $5 \mathrm{~min}$ to remove tissue residues, and then, $500 \mu \mathrm{l}$ of the

180 centrifuged volume was used for RNA extraction using $1 \mathrm{~mL}$ of TRIzol reagent (Life

181 Technologies, Thermo Fischer, USA). Treatment with DNase I (Ambion, Thermo

182 Fischer, USA) was performed to prevent contamination by genomic DNA. cDNA was

183 synthesized using a cDNA High Capacity Kit (Applied Biosystems, Thermo Fischer,

184 USA) according to the manufacturer's instructions by subjecting the samples to the

185 following cycle: $25^{\circ} \mathrm{C}$ for $10 \mathrm{~min}, 37^{\circ} \mathrm{C}$ for $120 \mathrm{~min}$ and $85^{\circ} \mathrm{C}$ for $5 \mathrm{~min}$. qPCR was

186 performed using a StepOnePlus Real-Time qPCR system, TaqMan Master Mix

187 Reagents (Applied Biosystems, Thermo Fischer, USA) and primers and probes specific

188 for the protein E sequence (38). Samples were then subjected to the following cycle:

$18950^{\circ} \mathrm{C}$ for $2 \mathrm{~min}$, followed by 40 cycles of $95^{\circ} \mathrm{C}$ for $10 \mathrm{~min}, 95^{\circ} \mathrm{C}$ for $15 \mathrm{sec}$, and $60^{\circ} \mathrm{C}$

190 for $1 \mathrm{~min}$.

\subsubsection{RT-qPCR}

The placenta was macerated in $1.5 \mathrm{~mL}$ of TRIzol reagent (Life Technologies,

193 Thermo Fischer, USA). RNA extraction was performed following the manufacturer's

194 protocol. cDNA was prepared using Power SYBR Green PCR Master Mix (Life

195 Technologies, Thermo Fisher, USA). The reaction was carried out for selected genes

196 using intron-spanning primers (Table 1) and the StepOnePlus Real-Time PCR system

197 (Life Technologies, Thermo Fischer, USA). Samples were subjected to the following

198 cycle: $95^{\circ} \mathrm{C}$ for $10 \mathrm{~min}$, followed by 40 amplification cycles consisting of DNA

199 denaturation for $30 \mathrm{sec}$ at $95^{\circ} \mathrm{C}$ and annealing of primers for $30 \mathrm{sec}$ at $60^{\circ} \mathrm{C}$. The

200 threshold cycle $(\mathrm{Ct})$ was determined for each gene of interest and for the reference

201 genes glycerol 3-phosphate dehydrogenase (Gapdh) and RNA Polymerase II Subunit A

202 (Polr2a). The relative expression of each gene was calculated using $2^{-\Delta \Delta \mathrm{CT}}$ (39) and 
203 graphically expressed as the fold-increase. The efficiency was calculated using the

204 standard curve method. The melting curves were analyzed for each sample.

205 Table 1: Primer sequences for the real-time PCR assay.

\begin{tabular}{lll}
\hline Gene & Primer sequences & GenBank accession no. \\
\hline ZIKV & 5'CCGCTGCCCAACACAAG3' & \\
& 5'CCACTAACGTTCTTTTGCAGACAT3' & \\
\hline$I l 6$ & 5'TCATATCTTCAACCAAGAGGTA3' & NM_031168.2 \\
& 5'CAGTGAGGAATGTCCACAAACTG3' & \\
\hline$I l 1 b$ & 5'GTAATGAAAGACGGCACACC3' & XM_006498795.4 \\
& 5'ATTAGAAACAGTCCAGCCCA3' & \\
\hline Illo & 5'TAAGGGTTACTTGGGTTGCCAAG3' & NM_010548.2 \\
& 5'CAAATGCTCCTTGATTTCTGGGC3' & \\
\hline Ifng & 5'AGCAACAGCAAGGCGAAAA3' & NM_008337.4 \\
& 5'CTGGACCTGTGGGTTGTTGA3' & \\
\hline Ifn1 & 5'CTGGAGCAGCTGAATGGAAAG3' & NM_010510.1 \\
& 5'CTTGAAGTCCGCCCTGTAGGT3' & \\
\hline Gapdh & 5'CTTTGTCAAGCTCATTTCCTGG3' & XM_017321385.2 \\
& 5'TCTTGCTCAGTGTCCTTGC3' & \\
\hline Tnf & 5'CCTCACACTCAGATCATCTTCTCA3' & NM_013693.3 \\
& 5'TGGTTCTCTTTGAGATCCATGC3' & \\
\hline Polr2a & 5'TCTGCCAAGAATGTGACGCT3' & NM_001291068.1 \\
& 5'CCAAGCGGCAAAGAATGTCC3' & \\
\hline
\end{tabular}

2.4 Detection of cytokines and chemokines in maternal serum and the placenta

Initially, placental tissue was homogenized in extraction buffer $(50 \mathrm{mM}$ Tris,

$208150 \mathrm{mM} \mathrm{NaCl}, 1 \mathrm{X}$ Triton, $0.1 \%$ SDS, $5 \mathrm{mM}$ EDTA, $5 \mathrm{mM} \mathrm{NaF}, 50 \mathrm{mM}$ sodium

209 pyrophosphate, $1 \mathrm{mM}$ sodium orthovanadate, $\mathrm{pH}$ 7.4) containing complete protease

210 inhibitor cocktail (Roche Applied Science, Germany) with TissueLyser LT (Qiagen,

211 Germany). The protein concentration of each sample was analyzed using a Pierce ${ }^{\mathrm{TM}}$

212 BCA Protein Assay Kit (Thermo Scientific, USA) according to the manufacturer's

213 instructions. Analysis of the cytokines IL- 6 and IL-1 $\beta$ and the chemokines monocyte

214 chemoattractant protein-1 (MCP-1/CCL2) and chemokine (C-X-C motif) ligand 1

215 (CXCL1) in maternal serum and placenta was performed with MILLIPLEX MAP

216 Mouse Cytokine/Chemokine Magnetic Bead Panel - Immunology Multiplex Assays

217 (MCYTOMAG-70K, Merck Millipore, Germany) following the manufacturer's

218 recommendations. The plate with samples and magnetic beads was analyzed on a 
219 MAGPIX ${ }^{2}$ System (Merck Millipore, Germany). The analyses were performed using

220 Luminex xPonent $₫ \square$ for MAGPIX® $\square$ v software. 4.2 (Luminex Corp., USA). For

221 each reaction well, the MAGPIX Luminex ${ }^{\circledR}$ platform reports the median fluorescence

222 intensity (MFI) for each of the analytes in the sample. The levels of each analyte were

223 then calculated against the standard curve. The ratio between the value obtained and the

224 protein quantification for each sample was determined and plotted.

\subsection{Virus titration using plaque assays}

Blood from mock- and $\mathrm{ZIKV}_{\mathrm{PE} 243}$-infected mice was collected from the base of

the tail at 4 hours, 48 hours and 144 hours following the appropriate treatments and

subsequently centrifuged at $400 \mathrm{~g}$ for $30 \mathrm{~min}$ for plasma separation. Samples obtained at

different periods post infection were titrated using a plaque assay. Vero cells (obtained

231 from ATCC ${ }^{\circledR}$ CCL81 ${ }^{\mathrm{TM}}$ ) (African green monkey kidney epithelial cell line) were plated

232 in 24 -well plates at $4 \times 10^{4}$ cells per well in Dulbecco's modified Eagle's medium

233 (DMEM) (GIBCO, Thermo Fisher, USA) supplemented with 5\% fetal bovine serum

234 (FBS) (GIBCO, Thermo Fisher, USA) and 1\% gentamicin $(10 \mu \mathrm{g} / \mathrm{ml})(\mathrm{GIBCO}$, Thermo

235 Fisher, USA) and cultured overnight for complete adhesion at $37^{\circ} \mathrm{C}$ with $5 \% \mathrm{CO}_{2}$.

236 Then, the medium was removed, and the cells were washed with 1x PBS and incubated

237 with serial (base 10) dilutions of virus in FBS-free medium. After 90 min of incubation

238 under gentle shaking, the medium was removed, and the cells were washed with 1x PBS

239 and cultured with $1.5 \%$ carboxymethylcellulose (CMC) supplemented with $1 \%$ FBS

240 (GIBCO, Thermo Fisher, USA). After 5 days, the cells were fixed overnight with 4\%

241 formaldehyde and stained with $1 \%$ crystal violet in $20 \%$ methanol (ISOFAR, Brazil) for

2421 hour. Plaques were counted, and the virus yield was calculated and expressed as

243 plaque-forming units per milliliter (PFU/ml). 


\subsection{Histological, immunohistochemistry and TUNEL analyses of the placenta}

Placental fragments were fixed overnight and subjected to dehydration

246 (increasing ethanol series; ISOFAR, Brazil), diaphanization with xylol (ISOFAR,

247 Brazil) and paraffin (Histopar, Easypath, Brazil). Sections $(5 \mu \mathrm{m})$ were prepared using a

248 Rotatory Microtome CUT 5062 (Slee Medical GmbH, Germany) and subjected to

249 immunohistochemistry and TUNEL analyses.

For immunohistochemistry, blocking of endogenous peroxidase was performed

251 with $3 \%$ hydrogen peroxide diluted in PBS, followed by microwave antigenic recovery

252 in Tris-EDTA $(\mathrm{pH}=9)$ and sodium citrate $(\mathrm{pH}=6)$ buffers $(15 \mathrm{~min}$ for Tris-EDTA buffer

253 and 8 min for citrate buffer). Sections were washed in PBS + 0.2\% Tween and exposed

254 to $3 \%$ PBS/BSA for 1 hour. Sections were then incubated overnight at $4^{\circ} \mathrm{C}$ with the

255 following primary antibodies: anti-Ki-67 (1:100 - [M3064]; Spring Bioscience, USA),

256 anti-P-gp (1:500 - Mdr1[sc-55510]; Santa Cruz Biotechnology, USA), anti-Bcrp (1:100

257 - Bcrp [MAB4146]; Merck Millipore, USA), anti-Abcg1 (1:100 - [PA5-13462];

258 Thermo Fisher Scientific, USA) or anti-Abca1 (1:100 - [ab18180]; Abcam Plc, UK).

259 The next day, sections were incubated with the biotin-conjugated secondary antibody

260 SPD-060 (Spring Bioscience, USA) for 1 hour at room temperature. Three washes were

261 performed with PBS $+0.2 \%$ Tween followed by incubation with streptavidin (SPD-060

262 - Spring Bioscience, USA) for $30 \mathrm{~min}$. Sections were stained with 3,3-diamino-

263 benzidine (DAB) (SPD-060 - Spring Bioscience, USA), counterstained with

264 hematoxylin (Proquímios, Brazil), dehydrated, diaphanized and mounted with a

265 coverslip and Entellan (Merck, Germany).

266 For analysis of apoptotic nuclei, terminal deoxynucleotidyl transferase dUTP 267 nick-end labeling (TUNEL) staining was performed using an ApopTag® In Situ

268 Peroxidase Detection Kit (S7100, Merck Millipore, USA) according to the 
269 manufacturer's recommendations and as previously described (36). All negative controls

270 were prepared with omission of the primary antibody.

271 Image acquisition was performed using a high-resolution Olympus DP72

272 (Olympus Corporation, Japan) camera coupled to an Olympus BX53 light microscope

273 (Olympus Corporation, Japan). For nuclear quantification of Ki-67 and TUNEL

274 immunolabeling, Stepanizer software (40) was used. For this analysis, we evaluated 15

275 images from different random fields of the Lz (labyrinth zone) and Jz (junctional zone)

276 for each animal, in a total of five animals from each ICompetent group and three

277 animals from each ICompromised group. A total of 360 digital images (40X) randomly

278 captured per placental region ( $\mathrm{Lz}$ and $\mathrm{Jz}$ ) were evaluated in each experimental group.

279 The total number of immunolabeled Ki-67 or TUNEL nuclei in each digital image was

280 normalized by the total image area to obtain an index of the estimated number of

281 proliferative and apoptotic nuclei in the entire histological section analyzed. Analysis

282 was undertaken by two investigators blinded to the treatment.

283 Quantification of P-gp, Bcrp, Abca1 and Abcg1 staining was performed using 284 the Image-Pro Plus, version 5.0 software (Media Cybernetics, USA) mask tool. The 285 percentage of viable tissue area was considered upon exclusion of negative spaces. A 286 total of 360 digital images (40X) randomly captured per placental region ( $\mathrm{Lz}$ and $\mathrm{Jz}$ )

287 were evaluated in each experimental group. Analysis was undertaken by two 288 investigators blinded to the treatment.

\subsection{Transmission electron microscopy (TEM)}

290 Sections of the placental Lz and Jz were fixed in paraformaldehyde 4\% (Sigma-Aldrich,

291 Brazil) for 48 hours, postfixed with osmium tetroxide (Electron Microscopy Sciences,

292 USA) and potassium ferrocyanide (Electron Microscopy Sciences, USA) for 60 min and 293 dehydrated with an increasing series of acetone $(30 \%, 50 \%, 70 \%, 90 \%$ and two of 
294 100\%) (ISOFAR, Brazil). Sections were subsequently embedded with EPOXI resin

295 (Electron Microscopy Sciences, USA) and acetone (1:2, 1:1 and 2:1, respectively).

296 After polymerization, ultrafine sections $(70 \mathrm{~nm})$ were prepared (Leica Microsystems,

297 USA) and collected into 300 mesh copper grids (Electron Microscopy Sciences, USA).

298 Tissue was contrasted with uranyl acetate and lead citrate and visualized using a JEOL

299 JEM-1011 transmission electron microscope (JEOL, Ltd., Akishima, Tokyo, Japan).

300 Digital micrographs were captured using an ORIUS CCD digital camera (Gatan, Inc.,

301 Pleasanton, California, EUA) at 6000× magnification. An overall qualitative analysis of

302 the $\mathrm{Lz}$ and $\mathrm{Jz}$ in different groups was performed by investigating the ultrastructural

303 characteristics of the mitochondria and the ER cisterns. The qualitative evaluation

304 consisted of analyzing disruption of the mitochondrial membranes, mitochondrial

305 morphology, preservation of mitochondrial cristae and matrix intensity (41).

306 Ultrastructural analysis of nuclear morphology and the presence of microvilli in

307 trophoblast sinusoidal giant cells was also undertaken. Analysis of ER cisterns was

308 performed by evaluating the dilation of their lumen (42).

$309 \quad 2.8$ Statistical analysis

310 GraphPad Prism 8 software (GraphPad Software, Inc., USA) was used for

311 statistical analysis. A D'Agostino \& Pearson normality test was used to evaluate normal

312 distribution, and outliers were identified using a Grubbs test. The data are expressed as

313 the mean $\square \pm$ SEM or individual values. One-way ANOVA followed by Tukey's posttest

314 was used for comparisons between different inbred groups, whereas Student's t-test or a

315 nonparametric Mann-Whitney test was performed to compare the outbred groups.

316 Differences were considered significant when $\mathrm{p}<0.05$. Pregnancy parameters were

317 evaluated using the mean value of all fetuses and placentae in a litter per dam and not

318 the individual conceptus, i.e., the mean value. In Figures 1 and 2, "n" represents the 
319 number of dams. For MET and immunostaining data, placentae closest to the mean

320 weight of all placentae were selected from each litter; "n" represents the number of

321 litters (35-37).

\section{Results}

3233.1 Weight gain during pregnancy is dependent on maternal immune status in ZIKV324 infected mice.

325 To determine the effect of ZIKV infection on fetal and placental phenotypes at 326 term (GD18.5), we infected ICompetent C57BL/6 and immunocompromised 327 (ICompromised) A129 mice with ZIKV at GD12.5 (Figure 1A). Given the very distinct 328 susceptibility of C57BL/6 and A129 mice to ZIKV, systemic infection models were 329 established by injecting high $\left(5 \times 10^{7} \mathrm{PFU}\right)$ and low $\left(10^{3} \mathrm{PFU}\right)$ virus inoculum titers. As

330 shown in Figure 1B, ICompetent C57BL/6 mice in all groups and ICompromised A129

331 dams inoculated with mock and low ZIKV titers exhibited higher maternal weight at 332 GD18.5 than at GD12.5 and GD0.5 (p<0.05). On the other hand, ICompromised A129 333 mice presented significant weight loss at GD18.5, despite showing an increase at 334 GD12.5 in relation to GD0.5 (Figure 1B).

3353.2 Immunocompetent and immunodeficient mice have distinct term placental and 336 fetal phenotypes in response to high and low ZIKV titer challenges in mid-pregnancy.

337 The fetuses from C57BL/6 dams and sires were called ICompetent. The fetuses 338 from the mating of A129 dams and C57BL/6 sires were called ICompromised. High339 ZIKV ICompetent mice exhibited 34\% fetal loss, whereas high-ZIKV-A129 mice had $340100 \%$ fetal loss (Figure 1C). In the low-ZIKV groups, C57BL/6 mice had no (0\%) fetal 341 death, while A129 mice exhibited a 42\% fetal death rate (Figure 1C). Fetal and fetal 342 head sizes were decreased in A129 mice compared to those in C57BL/6 dams infected 
343 with the high ZIKV titer ( $\mathrm{p}=0.05$; Figure $1 \mathrm{D}-\mathrm{G})$. However, no changes in fetal weight

344 or fetal head size were observed when the mice were infected with the low ZIKV titer

345 (Figure 1 D-G).
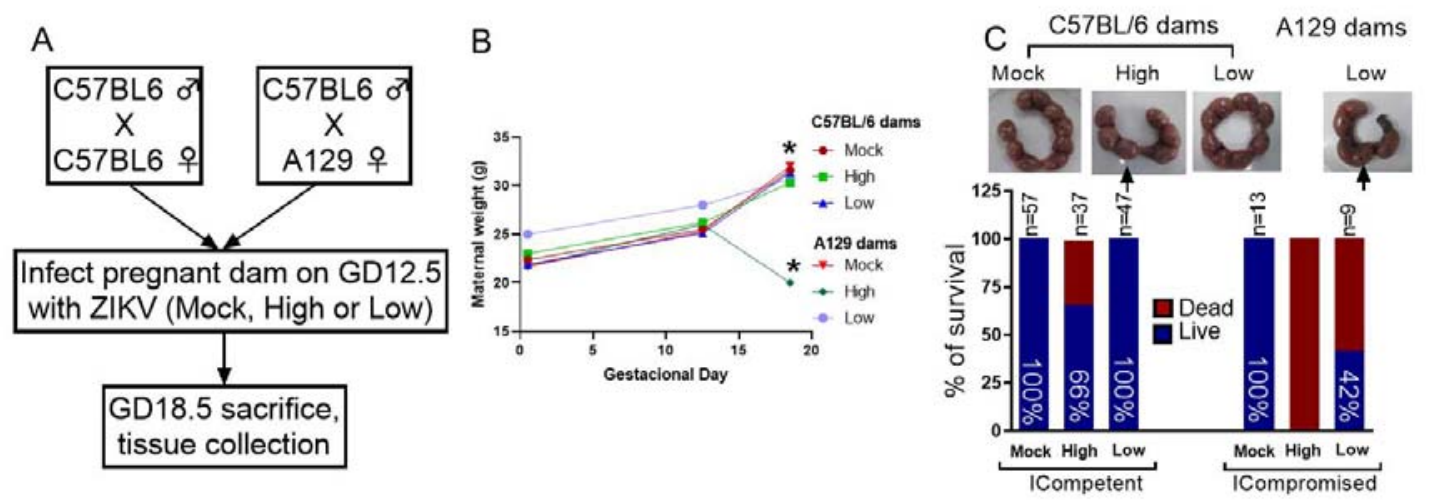

346
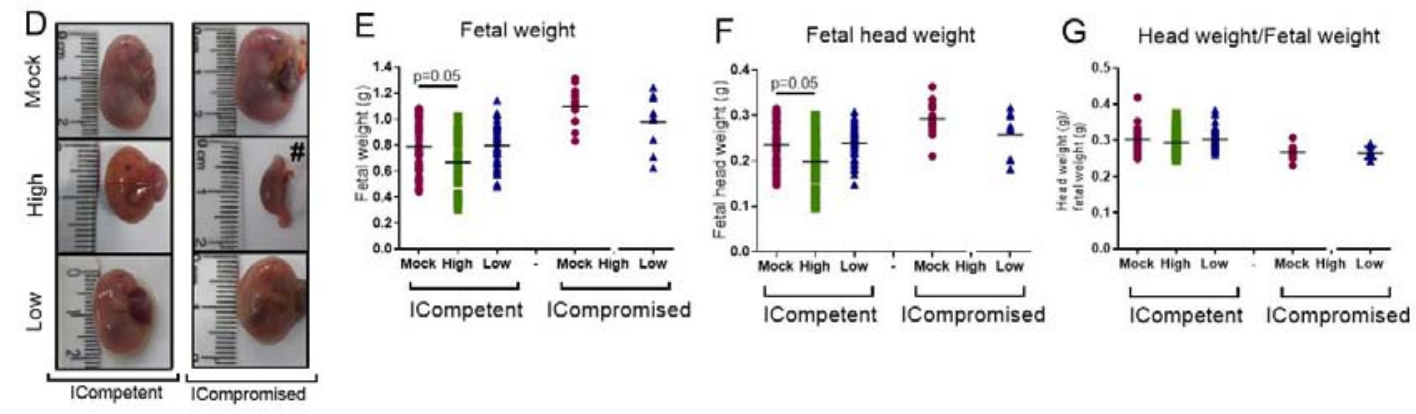

347 Figure 1: ZIKV infection induced fetal changes during pregnancy. A) Experimental 348 design: matings of C57BL/6 dams x C57BL/6 sires (mock $n=11$ dams; high ZIKV $n=15$ dams; low ZIKV n=9 dams) and A129 dams x C57BL/6 sires ( $\mathrm{n}=3$ dams/group). The mock control consisted of noninfected C6/36 cell supernatants; high ZIKV titers consisted of $5 \times 10^{7}$ plaque-forming units (PFU) of $\mathrm{ZIKV}_{\mathrm{PE} 243}$, and low ZIKV titers consisted of $10^{3} \mathrm{PFU}$ of $\mathrm{ZIKV}_{\mathrm{PE} 243}$. Fetuses from C57BL/6 dams and sires were termed ICompetent, whereas fetuses from A129 dams and C57BL/6 sires were termed ICompromised. B) Maternal weight gain throughout pregnancy, $*=p<0.05$, one-way ANOVA. C) Uterine horn and survival rates following ZIKV exposure (arrows show resorption sites). D) Fetal/reabsorption images (\#=example of fetal reabsorption). E) Fetal weight, F) fetal head weight and G) fetal head weight/fetal weight ratio. One-way ANOVA followed by Tukey's posttest was used to assess changes among ICompetent groups, whereas an unpaired Student's t-test or nonparametric Mann-Whitney test was used to assess differences between ICompromised groups. Values are the mean of individual plotted values.

\subsection{ZIKV is detected in the fetal brain of ICompromised, but not ICompetent mice.}


365 was also detected in the majority of the placentae of those mice (Figure 2A-B) but not

366 in the maternal and fetal C57BL/6 brains (Figure 2A-B), suggesting that the virus was

367 not transmitted to the fetuses. Although low ZIKV inoculation resulted in virus

368 detection in the spleens of pregnant C57BL/6 mice, infection was not evidenced in the

369 placentae or fetal brains. In contrast, ZIKV RNA was detected in all analyzed organs

370 from ICompromised A129 dams, including the maternal brain and spleen and the

371 placenta and fetal brain (Figure 2A-B). Viremia in maternal plasma was evaluated at 4,

37248 and 144 hours after infection. Within 4 hours, the presence of the virus was verified

373 in the serum (high ICompetent $=637.5 \mathrm{PFU} / \mathrm{mL}$, low ICompetent=740 PFU $/ \mathrm{mL}$ and low

374 ICompromised=325 PFU $/ \mathrm{mL}$ ), indicating that the virus was correctly inoculated.

375 Afterwards, ZIKV RNA was detected in ICompromised dams at 48 and 144 hours post

376 inoculation but not in ICompetent dams (Figure 2C).
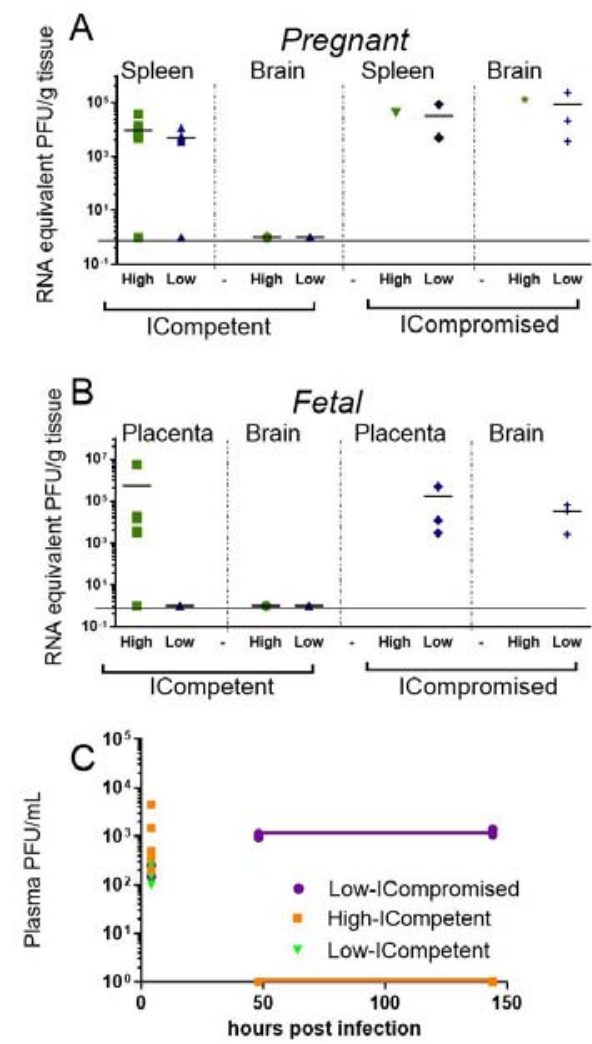
Figure 2: Viral load detection in maternal and fetal tissues after ZIKV infection. Pregnant ICompetent and ICompromised mice were inoculated i.v. with low and high doses of ZIKV. A-B) ZIKV RNA was measured via RT-qPCR in tissue samples obtained from maternal spleen and brain (A) and from placenta and fetal brain (B). C) The presence of ZIKV infectious particles in the plasma of infected dams was evaluated with a plaque assay 4,48 , and 144 hours post infection in ICompetent (mock $n=11$ dams; high ZIKV $\mathrm{n}=15$ dams; low ZIKV $\mathrm{n}=9$ dams) and ICompromised (mock $\mathrm{n}=3$ dams; high ZIKV n=3 dams; low ZIKV n=3 dams) mice.

\subsection{ZIKV infection induces distinct systemic and placental inflammatory responses in}

\section{ICompetent and ICompromised mice.}

388

The maternal serum and placental protein levels of specific cytokines and chemokines related to fetal death and preterm delivery (43-45) were evaluated to probe whether midgestation ZIKV infection would induce a maternal inflammatory response at term in our two distinct models. Since A129 infected with $10^{7}$ ZIKV-PFU showed $100 \%$ fetal loss, we proceeded using $10^{7} \mathrm{PFU}$ inoculation in C57BL/6 mice and $10^{3} \mathrm{PFU}$ inoculation in both C57BL/6 and A129 mice.

CCL2 was elevated in the serum of low-ZIKV ICompetent mice, but no other alteration was systemically detected in any ICompetent mice at this time point (Figure 3A-B). On the other hand, ICompromised dams showed significantly increased CXCL1 and IL-6 levels in the serum and a strong trend for an enhancement in CCL2 (Figure 3C). Analysis of cytokine and chemokine expression in the placenta demonstrated that the CXCL1 and CCL2 chemokines were also upregulated in ICompromised but not ICompetent mice (Figure 3D-F). Surprisingly, IL-6 protein expression was augmented in some of the low-ZIKV ICompetent mice $(58 \%$; $\mathrm{p}<0.05)$ but not in high-ZIKV mice or ICompromised mice (Figure 3D-F).

We also assessed the placental mRNA expression of a range of cytokines related to placental infective responses: Ifng, Il6, Ifnl, Tnf, Illb and Il10. All the infected mice showed a significant increase in $\operatorname{Tn} f$ mRNA expression, with higher levels detected in 
406 ICompetent mice (Figure 3G-I). The low-ZIKV ICompetent mice presented modest but

407 significant Ifnl expression, which was not detected in the high-ZIKV group (Figure

408 3G). Additionally, both low-ZIKV-infected groups (ICompetent and ICompromised)

409 presented increased Ifng mRNA expression (Figure 3G and 3I, p< 0.05). Interestingly,

410 placental Il6 mRNA levels were only elevated in ICompromised pregnancies compared

411 to mock pregnancies ( $\mathrm{p}=0.05$ ) (Figure 3I). $I l 1 \mathrm{~b}$ and $I l 10$ remained unchanged in all

412 groups analyzed (Figure 3 G-I).
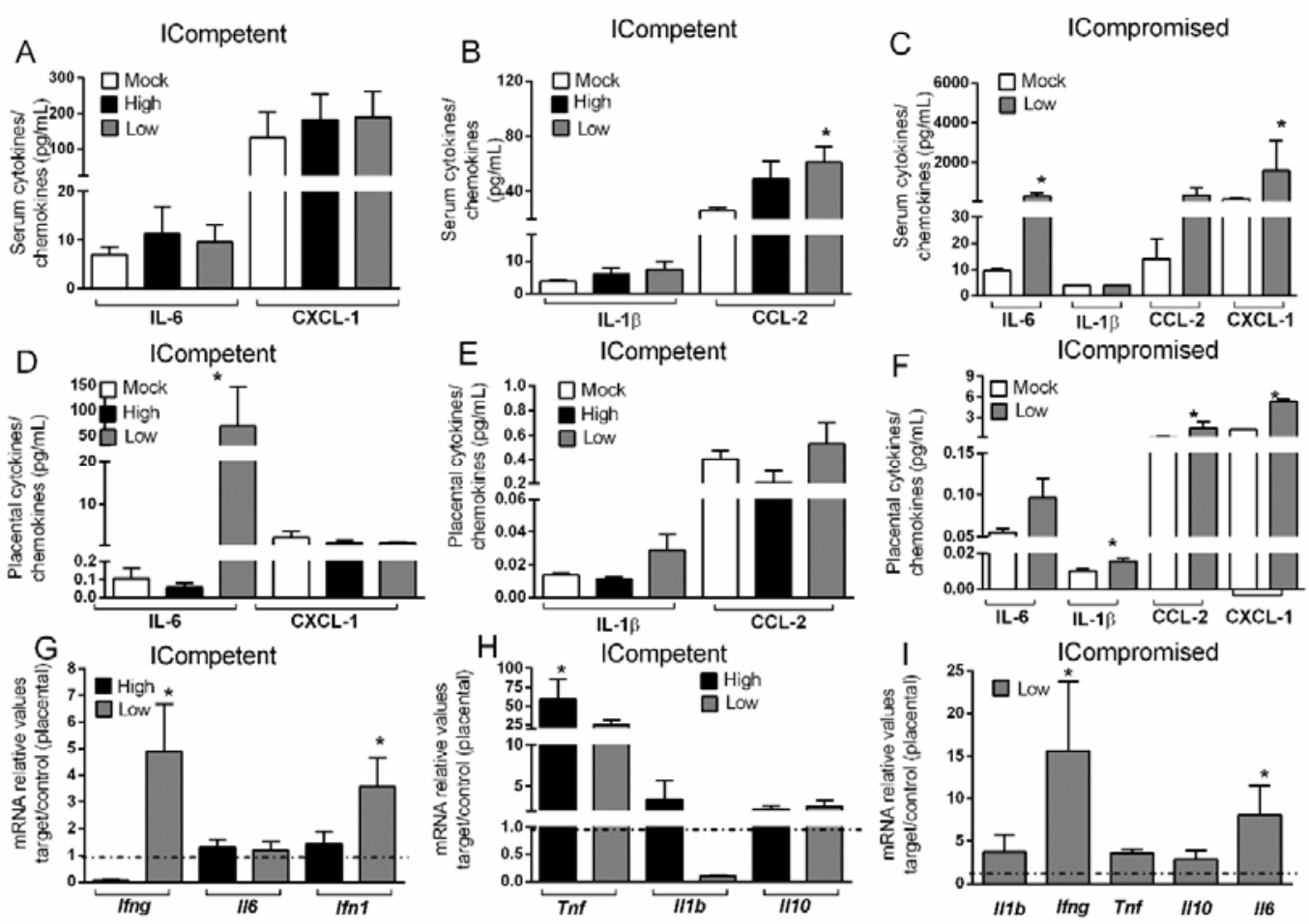

Figure 3: ZIKV during pregnancy promotes an inflammatory response in the maternal serum and in the placenta. A) Levels of IL-1 $\beta$, IL-6, CCL-2 and CXCL-1 in the maternal serum (A-C) and placenta (D-F) at GD18.5 in matings of C57BL/6 dams $\mathrm{x}$ C57BL/6 sires (ICompetent fetuses - mock $n=11$ dams; high ZIKV n=15 dams; low ZIKV $\mathrm{n}=9$ dams) and A129 dams x C57BL/6 sires (ICompromised fetuses $\mathrm{n}=3$ dams/group). Placental mRNA expression (G-I) of Ifng, Ifnl, Illb, Il6, Illo and Tnf. Broken lines show the expression levels in both lineages in the mock group. One-way ANOVA followed by Tukey's posttest was used to assess changes among ICompetent 
424 used to assess differences between ICompromised groups. The values are expressed as

the mean \pm SEM.

3.5 ZIKV affects placental proliferation, apoptosis and ultrastructure in a viral load-

and maternal immune status-dependent manner.

We did not observe changes in placental weight or in the fetal:placental weight

430 ratio in any of the groups investigated (data not shown). However, since we detected the

431 presence of ZIKV RNA in the placentas, we investigated the cellular proliferation (Ki-

$43267^{+}$cells) and apoptotic ratio in the $\mathrm{Lz}$ and $\mathrm{Jz}$ of the mouse placenta. Increased Ki-67

433 staining was observed in the Lz of high- and low-ZIKV-treated ICompetent animals

434 compared to mock animals (Figure 4A-D, $\mathrm{p}=0.005$ and $\mathrm{p}=0.015$ ), whereas the apoptotic

435 ratio in the $\mathrm{Lz}$ was increased in high-ZIKV dams and decreased in low-ZIKV dams

$436(\mathrm{p}=0.01$, Figure 4E-H). Although no differences were observed in Ki67 staining (Figure

437 5A-D), a similar apoptotic pattern was detected in the Jz of ICompetent pregnancies

$438(\mathrm{p}=0.008$ and $\mathrm{p}=0.004$, respectively; Figure 5E-H). In contrast, in ICompromised dams,

439 Lz Ki-67 staining was decreased ( $\mathrm{p}=0.001$; Figure 4I-L), while the apoptotic reaction

440 was increased in ZIKV-infected animals ( $\mathrm{p}=0.05$; Figure 4M-P). Jz from ICompromised

441 offspring exhibited increased Ki-67 labeling and no differences in the apoptotic reaction

$442(\mathrm{p}=0.001$; Figure $5 \mathrm{I}-\mathrm{L}$ and Figure 5M-P $)$. 

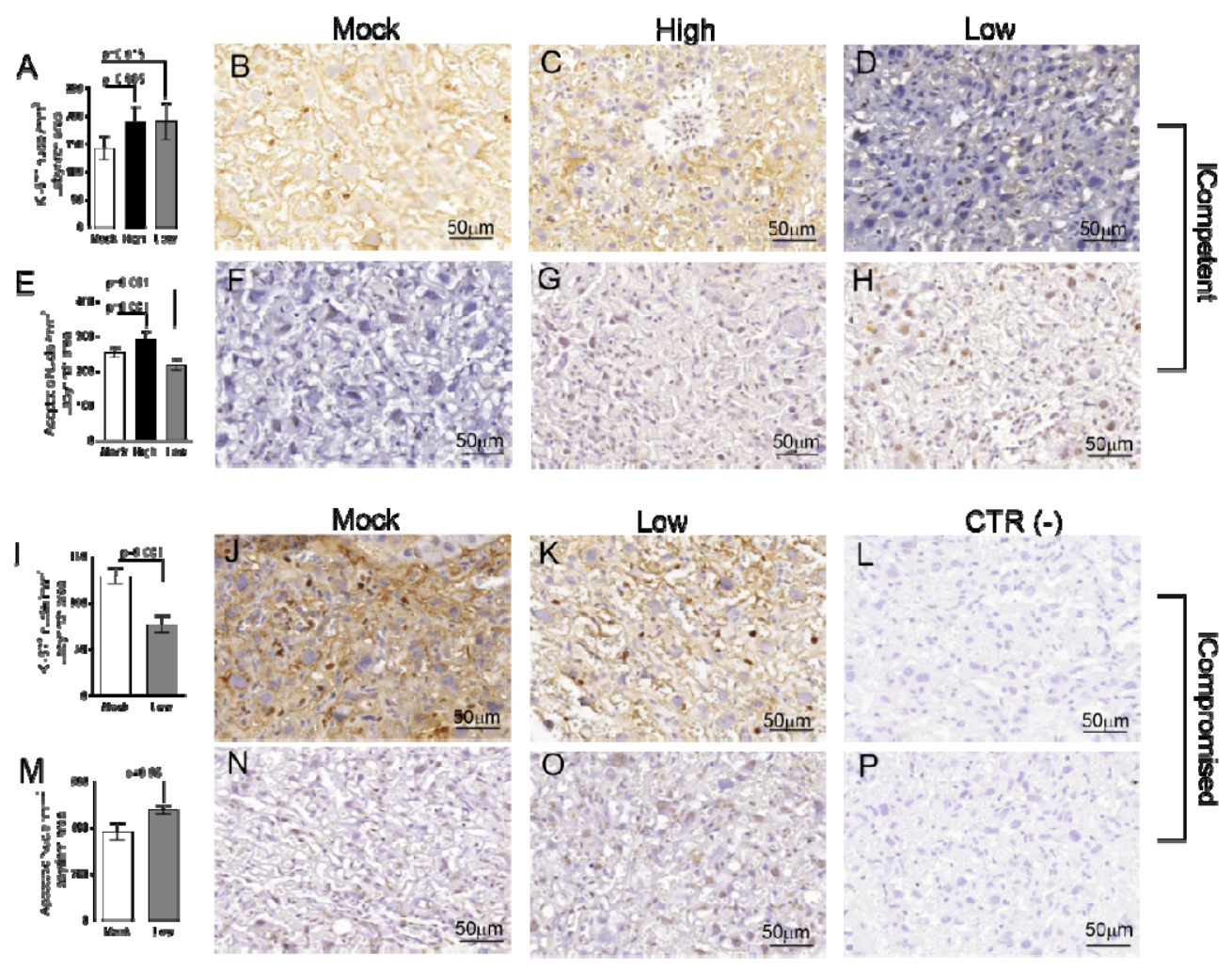

444 Figure 4: Labyrinthine remodeling in ICompetent and ICompromised placentae is

445 affected by gestational ZIKV infection. A total of 180 digital images (40X) randomly 446 captured from the whole labyrinth zone (Lz) of each placenta per dam were evaluated. 447 Immunolabeled nuclei from each digital image were quantified and normalized by the 448 total digital image area to obtain an index of the estimated number of proliferative and 449 apoptotic nuclei in the entire histological section. (A and I) Quantification and (B-D 450 and $\mathbf{J}-\mathbf{K})$ representative photomicrographs of $\mathrm{Ki}-67^{+}$stained nuclei in the $\mathrm{Lz}$ of 451 ICompetent ( $\mathrm{n}=6$ placentae from 6 independent dams/group) and ICompromised ( $\mathrm{n}=3$ 452 placentae from 3 independent dams/group) placentae, respectively. (E and $\mathbf{M}$ ) 453 Quantification and (F-H and N-O) representative photomicrographs of apoptotic nuclei 454 (TUNEL) in the Lz of ICompetent ( $n=5$ placentae from 5 independent dams/group) and 455 ICompromised ( $\mathrm{n}=3$ placentae from 3 independent dams/group) placentae, respectively. (L and P) Negative controls. One-way ANOVA followed by Tukey's posttest was used to assess changes among ICompetent groups, whereas an unpaired Student's t-test or nonparametric Mann-Whitney test was used to assess differences between ICompromised groups. The values are expressed as the mean \pm SEM. Images were captured at $40 \mathrm{X}$. Scale bar $=50 \mu \mathrm{m}$. 

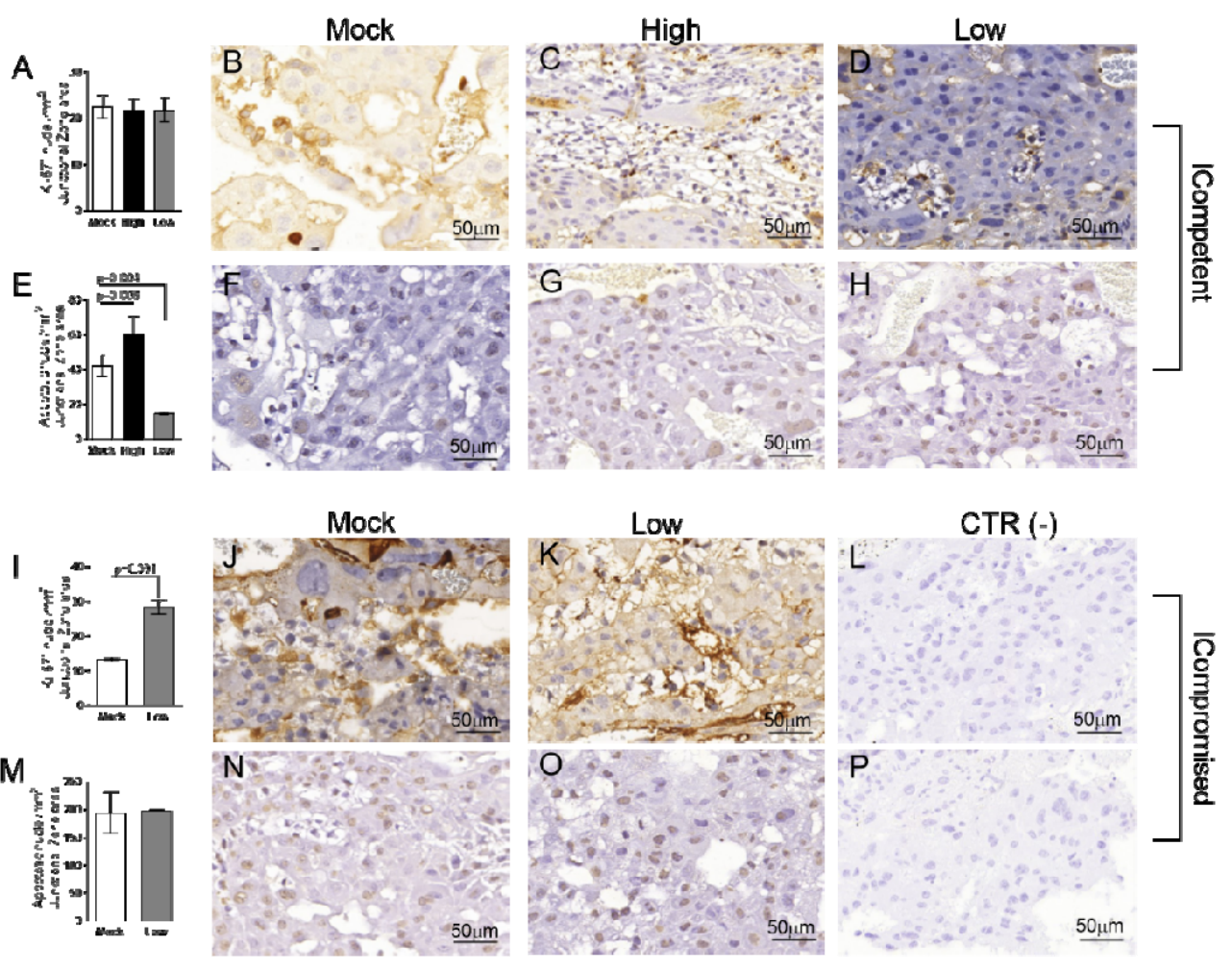

463

464

Figure 5: Junctional zone remodeling in ICompetent and ICompromised placentae

465 is affected by gestational ZIKV infection. A total of 180 digital images (40X) randomly captured from the whole junctional zone $(\mathrm{Jz})$ of each placenta per dam were evaluated. Immunolabeled nuclei from each digital image were quantified and normalized by the total digital image area to obtain an index of the estimated number of proliferative and apoptotic nuclei in the entire histological section. (A and I) Quantification and (B-D and J-K) representative photomicrographs of Ki-67 ${ }^{+}$stained nuclei in the $\mathrm{Jz}$ of ICompetent ( $\mathrm{n}=6$ placentae from 6 independent dams/group) and ICompromised ( $\mathrm{n}=3$ placentae from 3 independent dams/group) placentae, respectively. $(\mathbf{E}$ and $\mathbf{M})$ Quantification and (F-H and $\mathbf{N}-\mathbf{O})$ representative photomicrographs of apoptotic nuclei (TUNEL) in the $\mathrm{Jz}$ of ICompetent ( $\mathrm{n}=5$ placentae from 5 independent dams/group) and ICompromised ( $\mathrm{n}=3$ placentae from 3 independent dams/group) placentae, respectively. (L and P) Negative controls. One-way ANOVA followed by Tukey's posttest was used to assess changes among ICompetent groups, whereas an unpaired Student's t-test or nonparametric Mann-Whitney test was used to assess differences between ICompromised groups. The values are expressed as the mean \pm SEM. Images were captured at 40X. Scale bar $=50 \mu \mathrm{m}$.

\section{infection in ICompetent and ICompromised strains.}


nuclei, preserved mitochondrial ultrastructure and regular narrow ER cisternae (Figure

485 6A and 6B). In sharp contrast, high-ZIKV ICompetent (Figure 6C) infected placentae

486 showed fewer villi in the sinusoidal giant trophoblastic cells, degenerated mitochondria,

487 granular ER with dilated cisterns and euchromatic nuclei. The sinusoidal giant

488 trophoblastic cells in the low-ZIKV ICompetent mice (Figure 6D) also had fewer villi

489 and degenerated mitochondria than those in the mock placentae, but no effect on the ER

490 or euchromatic nuclei observed. Low-ZIKV ICompromised infected placentae (Figure

491 6E) showed fewer villi in the sinusoidal giant trophoblastic cells, degenerated

492 mitochondria, granular ER with dilated cisterns and euchromatic nuclei.
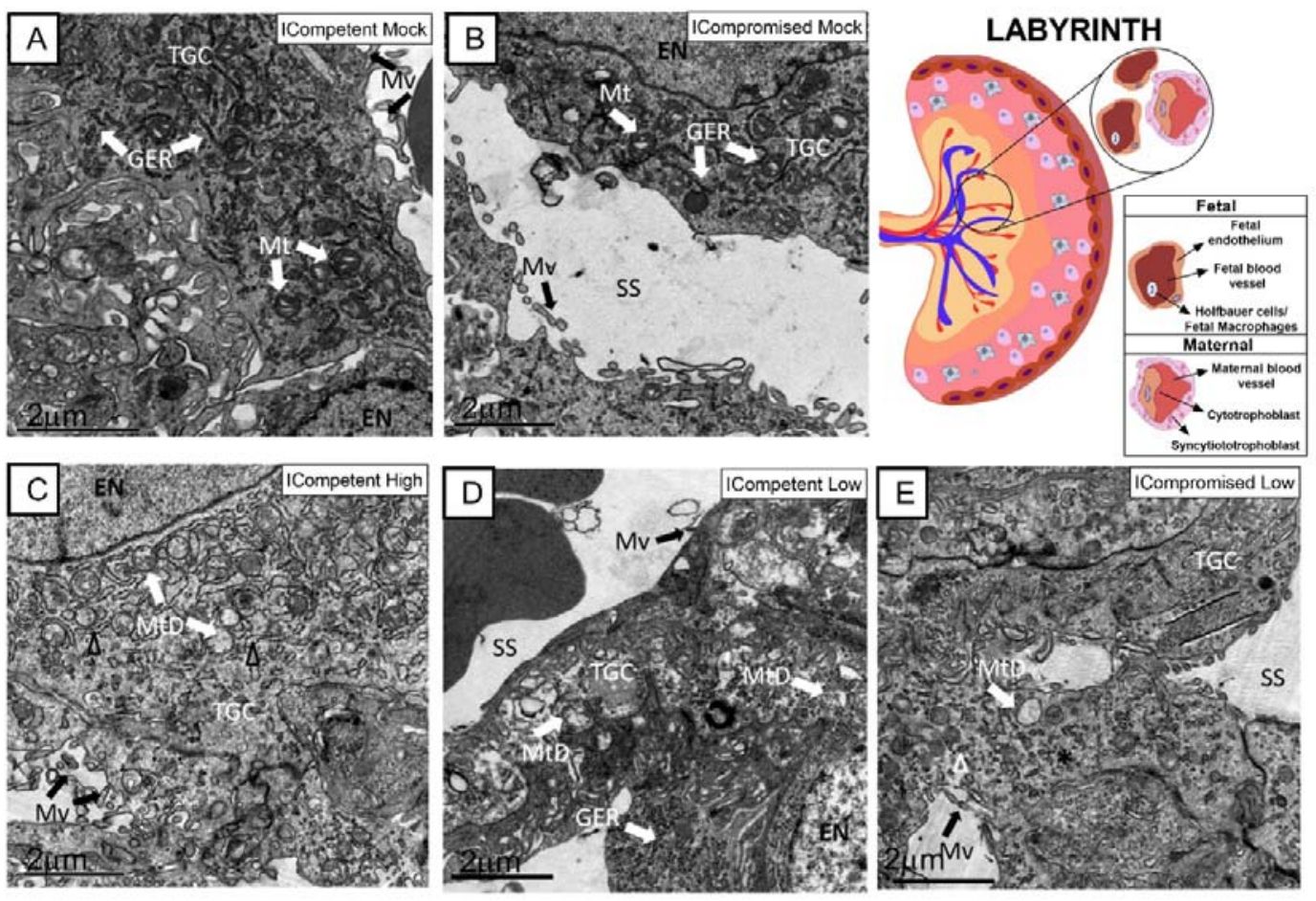

494 Figure 6: Associated ultrastructural changes in the placental Lz after ZIKV

495 infection. Transmission electron photomicrographs of ICompetent mock (A), 496 ICompromised mock (B), ICompetent high (C), ICompetent low (D) and 497 ICompromised low (E) groups ( $\mathrm{n}=5$ /group). We observed dilatation in the ER cisterns 498 of ICompetent high placentas. Additionally, there was a reduction in the microvilli in 499 both the ICompetent high and ICompetent low placentas. In the ICompromised low 500 group, we found fragmented ER and microvillus reduction. All infected groups showed 501 degenerate mitochondria. GER=granular endoplasmic reticulum; $\Delta=$ dilated granular 502 endoplasmic reticulum; *=fragmented granular endoplasmic reticulum; 
$503 \mathrm{Mt}=$ mitochondria; $\mathrm{MtD}=$ degenerate mitochondria; $\mathrm{Mv}=$ microvilli; $\mathrm{EN}=$ euchromatic 504 nuclei; $\mathrm{SS}=$ sinusoidal space; TGC=trophoblastic giant cell. Scale bar $=2 \mu \mathrm{m}$.

505 The Jz of mock ICompetent and ICompromised placentae (Figure 7A and 7B)

506 exhibited euchromatic nuclei, with evident heterochromatin, preserved mitochondria

507 and narrow cisternae in a granular ER. High-ZIKV ICompetent Jz had degenerated

508 mitochondria, granular ER with dilated cisterns and euchromatic nuclei (Figure 7C).

509 Low-ZIKV ICompetent (Figure 7D) placentae exhibited euchromatic nuclei with

510 evident heterochromatin, preserved mitochondria and narrow cisternae in a granular ER,

511 whereas low-ZIKV ICompromised placentae had degenerated mitochondria, granular

512 ER with dilated cisterns and euchromatic nuclei (Figure 7E).
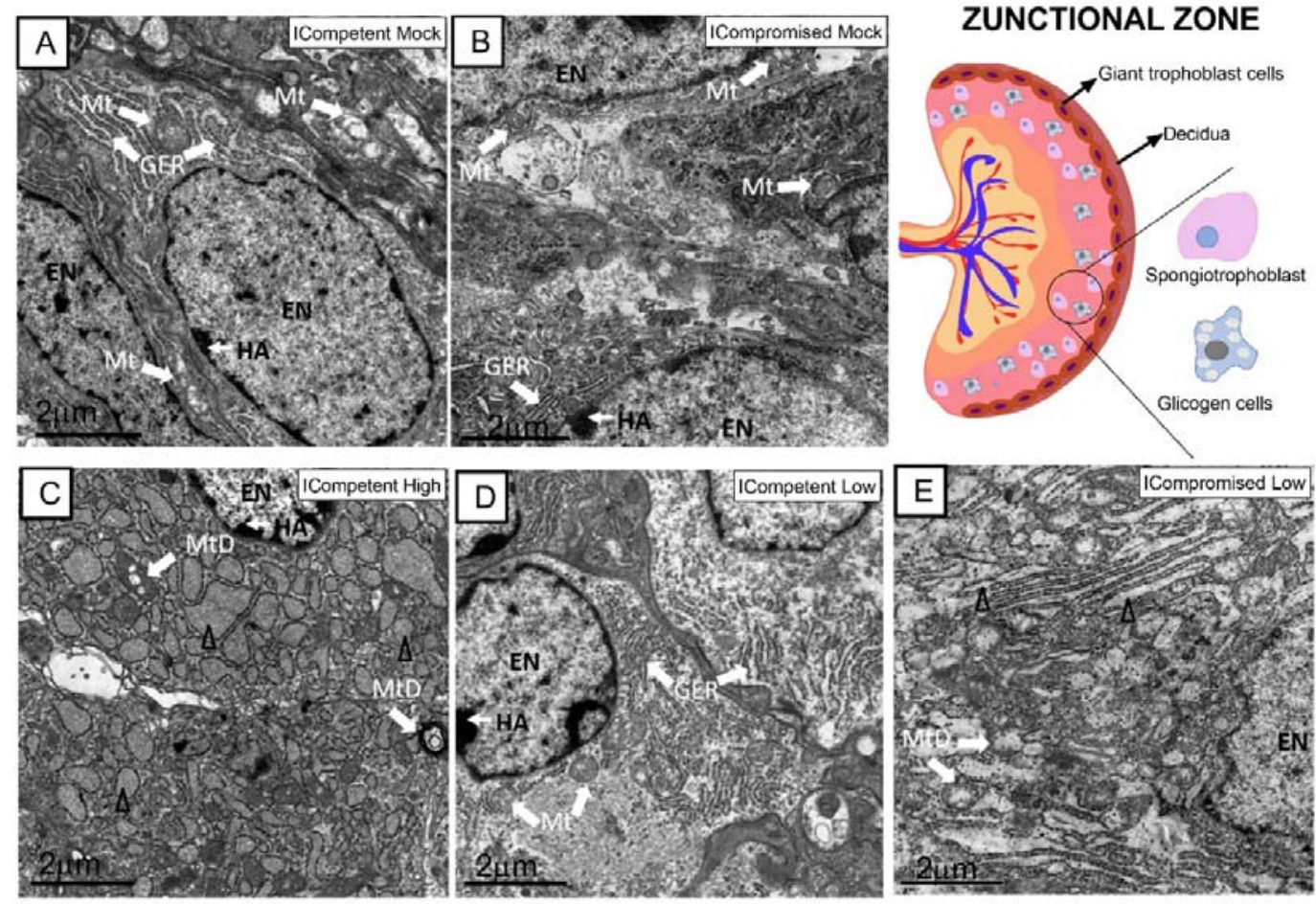

514 Figure 7: Associated ultrastructural changes in the placental Jz after ZIKV

515 infection. Transmission electron photomicrographs of ICompetent mock (A),

516 ICompromised mock (B), ICompetent high (C), ICompetent low (D) and

517 ICompromised low (E) groups ( $\mathrm{n}=5$ /group). We found deteriorating mitochondria and

518 dilated reticulum endoplasmic cisterns in both the high ICompetent and low

519 ICompromised groups. GER=granular endoplasmic reticulum; $\Delta=$ dilated granular

520 endoplasmic reticulum; $\mathrm{Mt}=$ mitochondria; $\mathrm{MtD}=$ degenerate mitochondria; 
$521 \mathrm{Mv}=$ microvilli; $\mathrm{EN}=$ =uchromatic nuclei; $\mathrm{SS}=$ sinusoidal space; $\mathrm{TGC}=$ trophoblastic giant 522 cell; $\mathrm{HA}=$ heterochromatin area. Scale bar $=2 \mu \mathrm{m}$.

5243.7 ZIKV differentially affects placental expression of drug and lipid ABC

\section{5 transporter systems.}

526 Evaluation of key ABC transporters in the Lz of mock and ZIKV-infected

527 ICompetent and ICompromised placentae revealed that immunolabeling of the drug P-

528 gp and Bcrp efflux transporter systems was primarily present at the cellular membranes

529 of the sinusoidal trophoblastic giant cells, with diffuse cytoplasmic Bcrp staining.

530 Labeling of the Abcal and Abcg1 lipid efflux transporters was moderately and

531 heterogeneously distributed within the Lz. Less Lz-P-gp was observed in ICompetent

532 mice infected with both high- and low-ZIKV infective regimens than in mock-treated

533 animals $(\mathrm{p}=0.001$ and $\mathrm{p}=0.002$, respectively; Figure $8 \mathrm{~A}-\mathrm{E})$, whereas reduced Bcrp and

534 Abcal staining was observed in high-ZIKV-infected mice $(\mathrm{p}=0.003$ and $\mathrm{p}=0.004$,

535 Figure 8F-J and Figure 8K-O, respectively). No changes in Abcg1 were observed in any

536 of the ICompetent experimental groups (Figure 8P-T). P-gp, Bcrp and Abca1

537 transporter immunostaining was downregulated in ICompromised low ZIKV-treated

538 animals $(\mathrm{p}=0.001, \mathrm{p}=0.05$ and $\mathrm{p}=0.05$, Figure 9A-D, Figure 9E-H and Figure 9I-L,

539 respectively). No changes in Abcg1 were observed in any of the ICompromised

540 experimental groups (Figure 9M-P). 

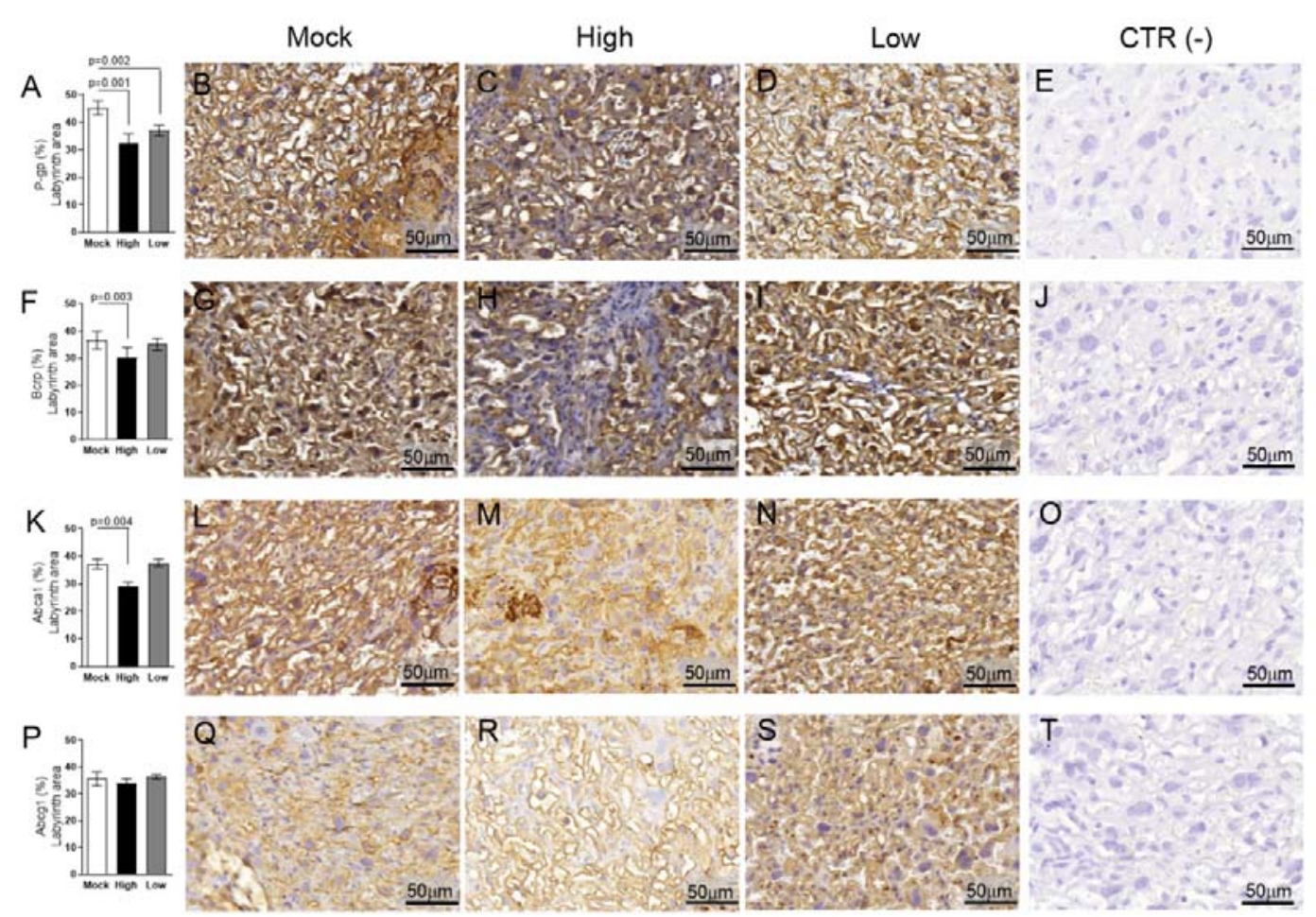

542 Figure 8: ZIKV infection decreases P-gp, Bcrp and Abca1 expression in the 543 placental $\mathbf{L z}$ of infected mice in the ICompetent groups. A total of 180 digital 544 images (40X) randomly captured from the whole labyrinth zone (Lz) of each placenta 545 per dam were evaluated. Immunolabeling in each digital image was quantified by 546 calculating the percentage area of the total stained labyrinthine tissue after exclusion of 547 the total negative space. (A) Quantification and (B-D) representative photomicrographs 548 of P-gp staining in the $\mathrm{Lz}$ of ICompetent $(\mathrm{n}=6$ placentae from 6 independent 549 dams/group) placenta. (F) Quantification and (G-I) representative photomicrographs of 550 Bcrp staining in the Lz of ICompetent ( $n=6$ placentae from 6 independent dams/group) 551 placenta. (K) Quantification and (L-N) representative photomicrographs of Abca1 552 staining in the Lz of ICompetent ( $\mathrm{n}=6$ placentae from 6 independent dams/group) 553 placenta. (P) Quantification and $(\mathbf{Q}-\mathbf{S})$ representative photomicrographs of Abcg1 554 staining in the $\mathrm{Lz}$ of ICompetent ( $\mathrm{n}=6$ placentae from 6 independent dams/group) 555 placenta. (E, J, O, T) Negative controls. One-way ANOVA followed by Tukey's post556 test. The values are expressed as the mean \pm SEM. Images were captured at 40X. Scale 557 bar $=50 \mu \mathrm{m}$. 

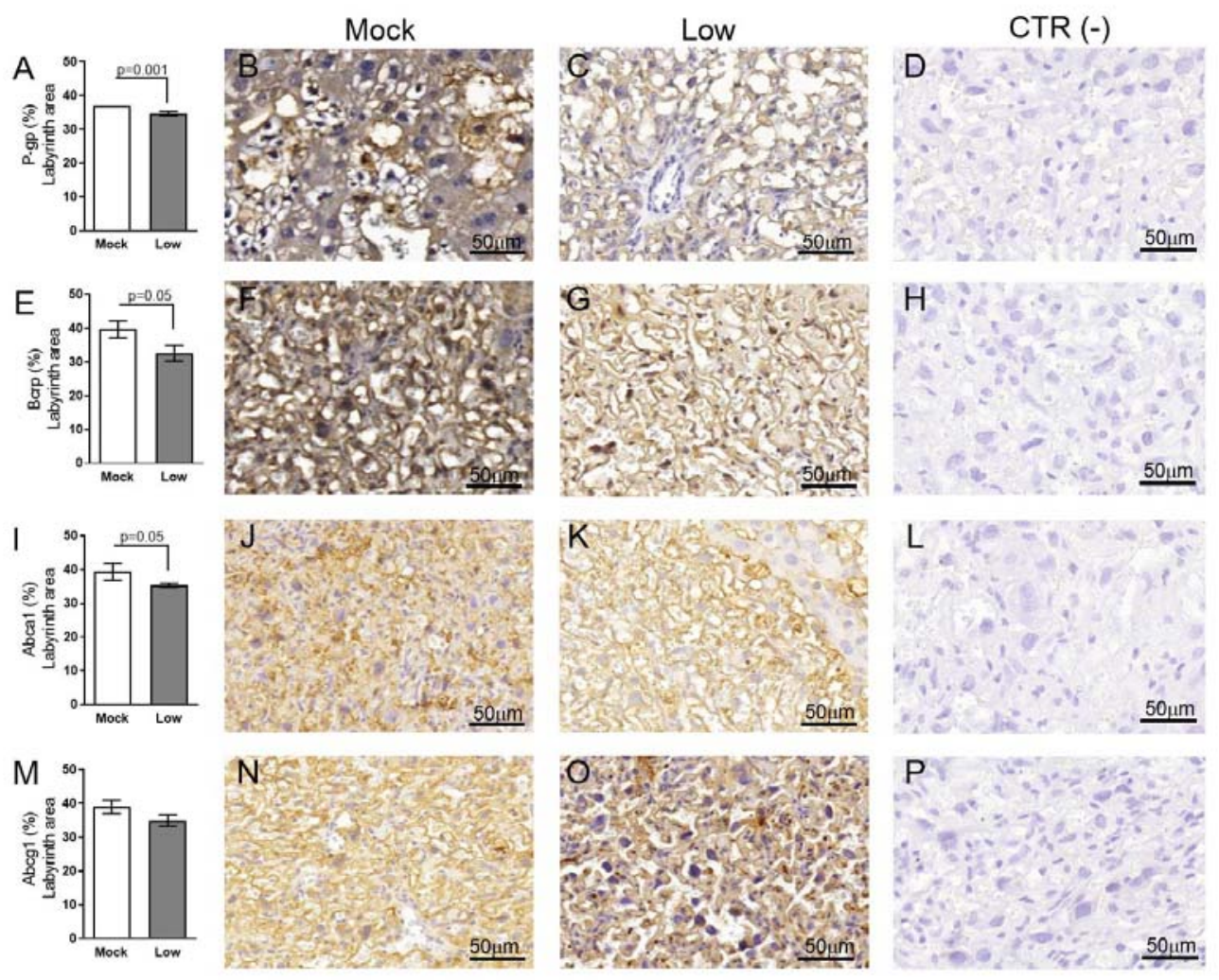

560 Figure 9: ZIKV infection decreases P-gp, Bcrp and Abca1 protein expression in 561 the placental $\mathbf{L z}$ of infected mice in the ICompromised groups. A total of 180 digital images (40X) randomly captured from the whole labyrinth zone (Lz) of each placenta per dam were evaluated. Immunolabeling in each digital image was quantified by calculating the percentage area of the total stained labyrinth zone tissue after exclusion of the total negative space. (A) Quantification and (B-C) representative photomicrographs of P-gp staining in the Lz of ICompromised ( $\mathrm{n}=3$ placentae from 3 independent dams/group) placenta. (E) Quantification and (F-G) representative photomicrographs of Bcrp staining in the Lz of ICompromised ( $\mathrm{n}=3$ placentae from 3 independent dams/group) placenta. (I) Quantification and (J-K) representative photomicrographs of Abcal staining in the Lz of ICompromised ( $\mathrm{n}=3$ placentae from 3 independent dams/group) placenta (M) quantification and (N-O) representative photomicrographs of Abcg1 staining in the Lz of ICompromised ( $\mathrm{n}=3$ placentae from 3 independent dams/group) placenta. (D, H, L, P) Negative controls. Unpaired Student's t-test or nonparametric Mann-Whitney test was used to assess differences between ICompromised groups. The values are expressed as the mean \pm SEM. Images were captured at $40 \mathrm{X}$. Scale bar $=50 \mu \mathrm{m}$.

579 endocrine layers of the mouse placenta) was assessed. P-gp and Bcrp were

580 predominantly localized at the cellular membranes of spongiotrophoblast cells, whereas

581 Abca1 and Abcg1 exhibited membrane and cytoplasmic staining. P-gp staining was 
582 decreased in Jz cells from the low-ZIKV ICompromised placentae $(\mathrm{p}=0.006)$, with no

583 other alterations observed (Figure 10A-T and Figure 11A-P).
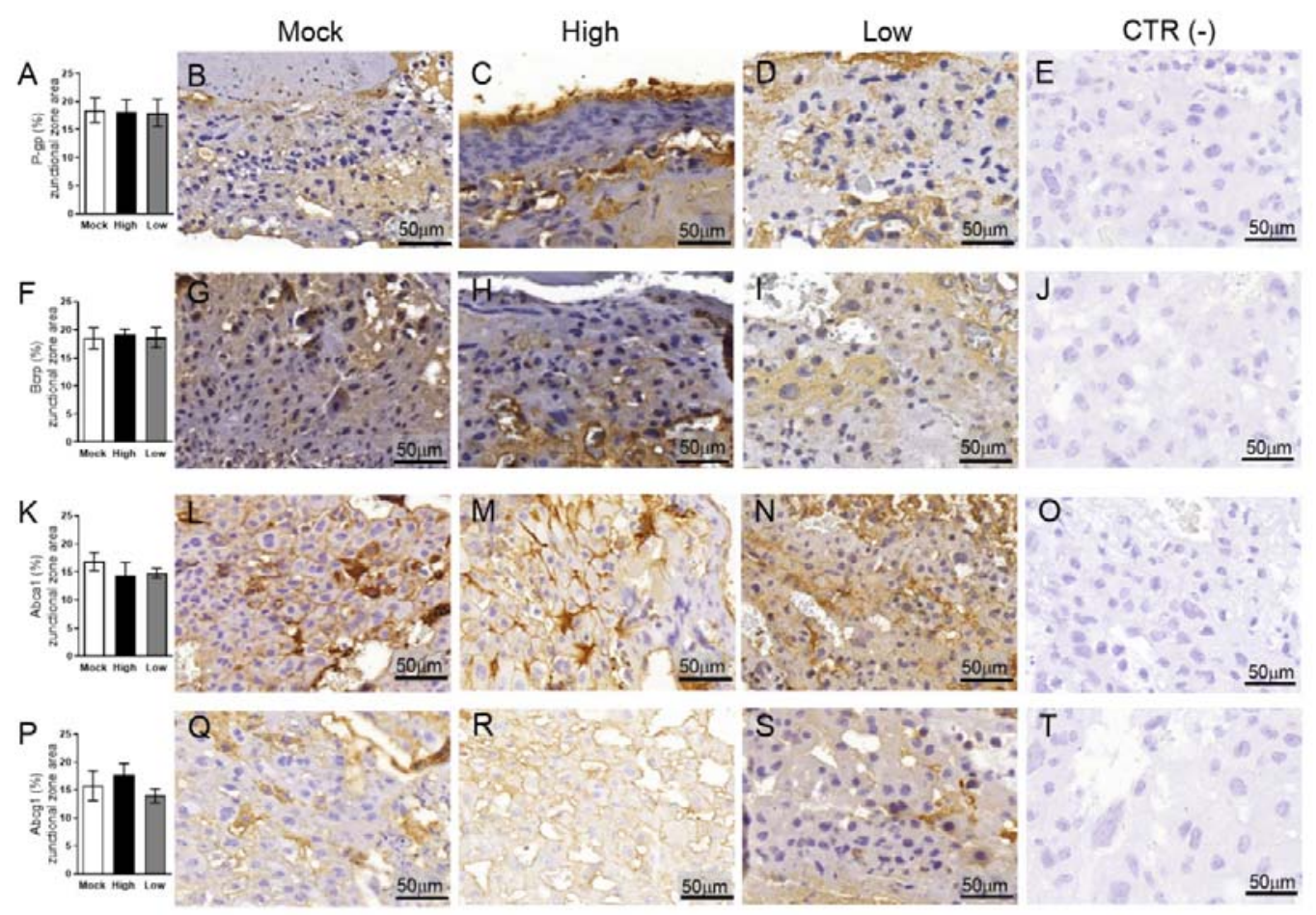

Figure 10: ZIKV infection did not impact P-gp, Bcrp, Abca1 or Abcg1 protein expression in the placental $\mathbf{~ J z}$ of infected mice in the ICompetent groups. A total of 180 digital images (40X) randomly captured from the whole junctional zone (Jz) of each placenta per dam were evaluated. Immunolabeling in each digital image was quantified by calculating the percentage area of the total stained junctional zone tissue after exclusion of the total negative space. (A) Quantification and (B-D) representative photomicrographs of P-gp staining in the $\mathrm{Jz}$ of ICompetent ( $\mathrm{n}=6$ placentae from 6 independent dams/group) placenta. (F) Quantification and (G-I) representative photomicrographs of Bcrp staining in the $\mathrm{Jz}$ of ICompetent $(\mathrm{n}=6$ placentae from 6 independent dams/group) placenta. (K) Quantification and (L-N) representative photomicrographs of Abcal staining in the $\mathrm{Jz}$ of ICompetent ( $\mathrm{n}=6$ placentae from 6 independent dams/group) placenta. (P) Quantification and (Q-S) representative photomicrographs of Abcg1 staining in the $\mathrm{Jz}$ of ICompetent ( $\mathrm{n}=6$ placentae from 6 independent dams/group) placenta. (E, J, O, T) Negative controls. One-way ANOVA

600 followed by Tukey's post-test. The values are expressed as the mean \pm SEM. Images

601 were captured at $40 \mathrm{X}$. Scale bar $=50 \mu \mathrm{m}$. 

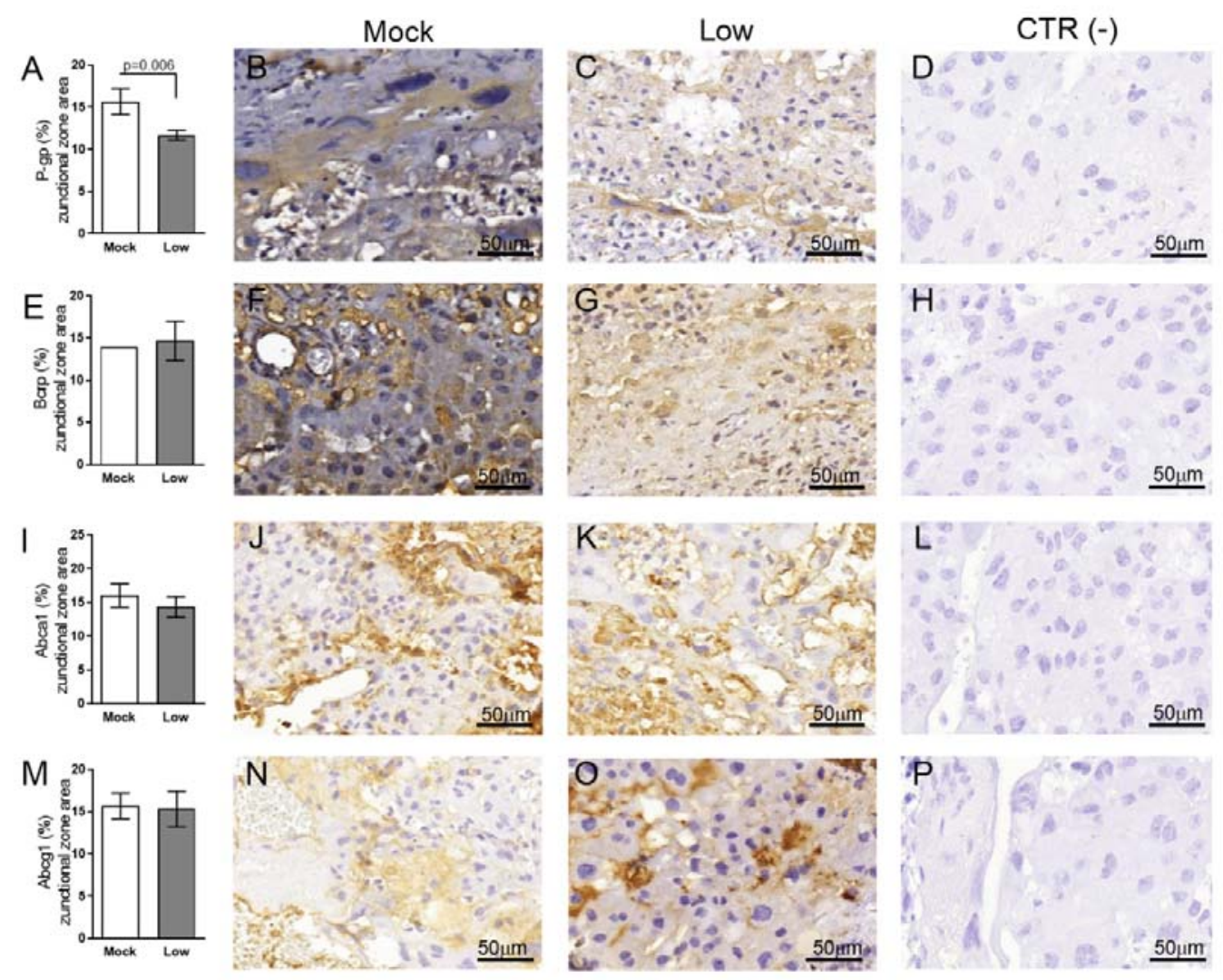

605

Figure 11: ZIKV infection decreases Bcrp protein expression in the placental Jz of

606 infected mice in the ICompromised group. A total of 180 digital images (40X)

607 randomly captured from the whole junctional zone $(\mathrm{Jz})$ of each placenta per dam were

608 evaluated. Immunolabeling in each digital image was quantified by calculating the percentage area of the total stained junctional zone tissue after exclusion of the total negative space. (A) Quantification and (B-C) representative photomicrographs of P-gp staining in the $\mathrm{Jz}$ of ICompromised ( $\mathrm{n}=3$ placentae from 3 independent dams/group) placenta. (E) Quantification and (F-G) representative photomicrographs of Bcrp staining in the $\mathrm{Jz}$ of ICompromised ( $\mathrm{n}=3$ placentae from 3 independent dams/group) placenta. (I) Quantification and (J-K) representative photomicrographs of Abca1 staining in the $\mathrm{Jz}$ of ICompromised ( $\mathrm{n}=3$ placentae from 3 independent dams/group) placenta. (M) Quantification and (N-O) representative photomicrographs of Abcg1 staining in the $\mathrm{Jz}$ of ICompromised ( $\mathrm{n}=3$ placentae from 3 independent dams/group) placenta. (D, H, L, P) Negative controls. Unpaired Student's t-test or nonparametric Mann-Whitney test was used to assess differences between ICompromised groups. The values are expressed as the mean \pm SEM. Images were captured at $40 \mathrm{X}$. Scale bar $=50$ $\mu \mathrm{m}$.

\section{Discussion}


626 at mid-pregnancy (GD12.5). Fetal survival rates, systemic and placental inflammatory

627 responses, placental ultrastructure and cell turnover, as well as the expression of key 628 drug (P-gp and Bcrp) and lipid (Abca1) efflux transporter systems in the placenta, were 629 consistently impacted by ZIKV in both strains. The magnitude of the effects was clearly

630 related to the infective titer (high and low) of ZIKV and maternal immune status 631 (ICompetent-C57BL/6 $\mathrm{x}$ and ICompromised-A129), and fetal alterations were not 632 exclusively dependent on virus detection in the fetuses.

633 Infection of ICompetent mice with ZIKV did not result in viremia in the initial 634 postinoculation phase, although viral RNA was detected in the maternal spleen in both 635 the high- and low-ZIKV groups, confirming systemic infection. This is consistent with a 636 previous report (46). Our data demonstrate that pregnant ICompetent C57BL/6 mice 637 were more susceptible to high ZIKV titers than to low ZIKV infective. Since viral RNA 638 was only detected in the placentae of high ZIKV-infected mice, fetal survival rates and 639 weights were impacted to a greater extent in those mice. Strikingly, even though the 640 virus was not present in the fetal brain (at least at term), fetal and fetal head weights 641 were lower in mice subjected to the high-ZIKV titer regimen, suggesting that high 642 infective viral load in mid-pregnancy, even in ICompetent individuals, can induce 643 IUGR and lower fetal head weight despite a lack of transmission to the fetal brain (47).

644 On the other hand, ICompromised placentae and fetal brains had detectable viral 645 transcripts, with no changes in weight, which is consistent with previous data (19). In 646 fact, in our models, the presence (ICompromised) or absence (ICompetent) of the virus 647 in the fetal brain did not correspond to fetal head size (decreased in only high 648 ICompetent). The data from ICompetent and ICompromised placentae demonstrate how 649 important the maternal immunological status is to control viremia, fetal survival and 650 accessibility of the virus to the fetal brain. The reason for the reduction in fetal brain 
651 size in C57BL/6 mice in the absence of fetal brain infection requires further

652 investigation. It is possible that fetal brains in the ICompetent mice may have been

653 exposed to ZIKV earlier in pregnancy, when viremia was present in the maternal blood,

654 and this may have severely compromised brain development. Of note, one limitation of

655 our study is that we measured fetal head weight instead of cortical thickness. Future

656 studies should investigate whether high- and low-ZIKV exposure alters cortical

657 thickness in ICompetent and ICompromised offspring.

658 A distinct inflammatory profile was also detected in the three analyzed groups.

659 At the protein level, low-ZIKV-ICompromised dams exhibited increased maternal IL-6

660 and CXCL-1 and placental CCL-2 and CXCL-1, whereas low-ZIKV-ICompetent dams

661 had increased maternal CCL2 and placental IL-6 levels. CCCL-2 and CXCL-1 are

662 related to fetal death and preterm delivery (43-45) and could be associated with

663 pronounced fetal injury detected upon ICompromised pregnancy. In addition, IL-6 was

664 previously demonstrated (48) to be related to fetal response syndrome, characterized by

665 activation of the fetal immune system. This syndrome is known to increase fetal

666 morbidity and affect several organs, such as the adrenal gland, brain and heart $(32,48-$

667 51). At the mRNA level, Il6 expression was only detected in ICompromised placentae

668 at term and may indicate a sustained harmful response in these mice until term. The IFN

669 signaling pathway may be triggered by ZIKV (17) and is one of the key mechanisms of

670 host defense and a viral target for immune evasion (20), but we only detected a slight

671 increase in Ifnl in low-ZIKV ICompetent mice at term. However, we cannot rule out

672 the possibility that these cytokines might have been produced earlier. Our findings

673 showed that Ifng expression was significantly enhanced in both ICompetent and

674 ICompromised low-ZIKV-derived placentae but not in high-ZIKV-infected mice.

675 Although we could not assess cytokine expression in high-ZIKV ICompromised 
676 placentas, one may extrapolate that low-ZIKV infection could result in stimulation of

677 Ifng producing cells, which has been previously shown to be protective for ZIKV-

678 infected mice (52).

679 Both ICompromised and ICompetent mice showed increased expression of

680 placental Tnf mRNA, which has been demonstrated to be directly related to placental

681 damage, abortion and premature birth (53-56). In addition, an increased $\operatorname{Tnf}$ response is

682 related to impaired placental hormone production and trophoblastic invasion and

683 increased apoptosis in pregnancy $(57,58)$. Although we did not assess TNF- $\alpha$ protein

684 levels in the placenta and maternal blood, this response could be implicated in the 685 overall damage detected.

686 Although differences in placental weight were not observed, ZIKV infection 687 mid-pregnancy had a profound effect on placental cellular turnover, dependent on titer, 688 strain and/or placental compartment. The $\mathrm{Lz}$ is responsible for fetal and maternal 689 nutrient, gas and waste exchange, while the Jz provides structural support, nutrient 690 storage and hormone synthesis (35). ZIKV induced a consistent increase in Lz 691 proliferation in all groups. However, the Lz apoptotic rate was increased only in the 692 high-ZIKV-ICompetent and ICompromised groups and decreased in low-ZIKV693 ICompetent mice. The mechanisms underlying these differences are unknown but may 694 be related to the distinct maternal and placental proinflammatory responses and/or to the 695 direct effect of the virus on the placenta (59). Increased Lz apoptosis in the high696 ICompetent-ZIKV group may be one of the mechanisms driving the lower fetal and 697 fetal head weight detected in this group. In this context, changes in placental turnover 698 can determine placental maturation and function and lead to fetal distress and 699 developmental abnormalities (60). An increase in the Lz apoptotic ratio may signify 700 damage to this placental layer, which is consistent with the fact that diverse pathological 
701 lesions associated with congenital disorders were described in placentae from women

702 infected by ZIKV at different stages of pregnancy (61). Conversely, no proliferative

703 changes were observed in the Jz in high-ZIKV and low-ZIKV ICompetent mice, while

704 increased and decreased apoptotic rates were detected. It follows that the lack of Jz-Ki-

70567 induction may suggest that this layer is less capable of restoring proliferation in

706 response to high-ZIKV challenge, and this may be related to decreased fetal growth.

707 Our placental ultrastructural analysis detected consistent differences across

708 ZIKV-exposed groups. The Lz and Jz layers from both strains exhibited signs of ER

709 stress, i.e., dilated ER cisterns or fragmented ER granular structures. These alterations

710 may result from the accumulation of folded or poorly folded viral proteins in the ER

711 lumen $(42,62,63)$. The Flaviridae family uses the ER to replicate $(64)$, and according to

712 Offerdahl et al. (2017)(63), there is evidence of ZIKV interacting with this organelle,

713 promoting an increased release of $\mathrm{Ca}^{+2}$ from the ER to the cell cytoplasm, thereby

714 causing an increase in the production of reactive oxygen species (ROS) $(65,66)$.

715 The mitochondrial ultrastructure in the Lz and $\mathrm{Jz}$ layers was severely impacted

716 by ZIKV exposure. We found evidence of mitochondrial degeneration, i.e.,

717 mitochondrial membrane rupture, absence of mitochondrial ridges and a less electron-

718 dense mitochondrial matrix, in all the treated groups. Placental mitochondrial

719 dysfunction is associated with IUGR $(67,68)$ and may be related, at least in part, to the

720 lower fetal weight observed in high-ZIKV-ICompetent fetuses along with the altered

721 placental apoptotic and proliferative patterns. Furthermore, mitochondrial dysfunction

722 together with ER stress is likely to modify the placental ROS balance and generate local

723 oxidative stress (69), which is associated with impaired fetal development (70). Of

724 importance, associations between mitochondrial disruption, ER stress and placental cell

725 senescence have been reported. Senescence is characterized as an irreversible 
726 interruption of the cell cycle and acquisition of a senescence-associated secretory

727 phenotype (SASP) that promotes the release of cytokines, such as IL-1, IL-6, IL-8 and

728 proinflammatory proteases (70). Therefore, the increased expression of IL-6 detected in

729 the placentas of ICompromised mice suggests a SASP profile, which may be related to

730 changes in the ER and mitochondrial ultrastructure, accompanied by important changes

731 in apoptosis and cell proliferation. The interactions between mitochondria and the ER

732 are critical for homeostasis and cell signaling (71). In conjunction with the ER,

733 mitochondria can regulate cell death mediators in response to hypoxia and inflammation

734 (72). The increase in apoptosis observed in the high-titer ICompetent groups and the

735 low-titer ICompromised group may be related to the mitochondrial damage and ER

736 stress observed. In fact, we observed an important decrease in microvillus abundance in

737 sinusoidal giant trophoblast cells. Previously, we observed a decrease in microvillus

738 density in the $\mathrm{Lz}$ of pregnancies exposed to malaria in pregnancy (MiP) (36). Together,

739 our data show that different gestational infective stimuli (MiP and ZIKV) are capable of

740 damaging placental microvillus abundance and impairing proper fetal-maternal

741 exchange function and fetal growth/survival.

742 Next, to investigate whether maternal ZIKV exposure may influence fetal

743 protection, we evaluated the placental localization and expression (semiquantitative) of

744 the ABC efflux transporter systems P-gp, Bcrp, Abcal and Abcg1, which are highly

745 enriched in labyrinthine microvilli and in human syncytiotrophoblasts. These efflux

746 transporters exchange drugs, environmental toxins, cytotoxic oxysterols and lipids

747 within the maternal-fetal interface (26). We found a consistent decrease in labyrinthine

748 P-gp expression in all ZIKV-exposed groups, demonstrating that ZIKV infection during

749 pregnancy has the potential to increase fetal exposure to P-gp substrates, such as

750 synthetic glucocorticoids, antibiotics, antiretrovirals, antifungals, stomach-protective 
751 drugs, and nonsteroidal anti-inflammatory drugs (26). Furthermore, Jz-P-gp was

752 decreased in ICompromised placentae. Although little is known about the function of

$753 \mathrm{ABC}$ transporters in the $\mathrm{Jz}$, our data highlight the need for further studies investigating

754 the biological importance of $\mathrm{ABC}$ transporters in the placental endocrine and structural

755 zones of the rodent hemochorial placenta under normal and infective conditions.

756 ZIKV impaired Lz Bcrp and Abca1 expression in ICompetent (high) and

757 ICompromised (low) mice. However, no effects were observed in ICompetent animals

758 at a low ZIKV titer or in Abcg1 in any experimental setting. Thus, ZIKV also likely

759 increases fetal accumulation of Bcrp substrates (antibiotics, antiretrovirals,

760 sulfonylureas, folate, mercuric species, estrogenic mycotoxins, carcinogens and

761 phototoxic compounds, among others) and disrupts placental lipid homeostasis (lipids,

762 cholesterol, and cytotoxic oxysterols) by reducing placental Abca1 expression $(26,73-$

763 76). We can speculate that the increased fetal accumulation of the P-gp, Bcrp and

764 Abca1 substrates during ZIKV infection may contribute to the establishment of

765 congenital Zika syndrome, although additional studies are clearly required to answer

766 this important question. The present data are in agreement with previous publications

767 showing that bacterial, viral and protozoan inflammation alters the expression and/or

768 function of P-gp, Bcrp and Abcal in biological barriers, such as the placenta, yolk sac

769 and blood-brain barriers $(26,27,36,64,77-79)$.

\section{5. Conclusion}

771 Our data show that gestational ZIKV impacts the fetal phenotype independently

772 of term fetal viremia. Abnormal placental cell turnover, ultrastructure and transporter

773 expression may result from specific proinflammatory responses that depend on the

774 ZIKV infective load and maternal immune status. Fetal accumulation of drugs, 
775 environmental toxins and lipids within the fetal compartment may potentially be

776 increased in ZIKV-infected pregnancies due to altered levels of key $\mathrm{ABC}$ transporters.

\section{Conflict of Interest:}

778 The authors declare that the research was conducted in the absence of any commercial

779 or financial relationships that could be construed as a potential conflict of interest.

\section{Author contributions}

781 CBVA, FFB, EB, LBA and TMOC conceived and designed the experiments. CBVA,

782 VRSM, SVAC, HRG, RPCS and VMON performed the experiments. CBVA, SVAC,

783 FFB, EB, SGM, LBA and TMOC analyzed the data. CBVA, VRSM, EB, LBA and

784 TMOC wrote the paper and edited the manuscript. All authors contributed to the article 785 and approved the submitted version.

\section{Funding}

787 This study was supported by the Bill \& Melinda Gates Foundation

788 (MCTI/CNPq/MS/SCTIE/Decit/Bill and Melinda Gates 05/2013; OPP1107597), the

789 Canadian Institutes for Health Research (SGM: Foundation-148368), Conselho

790 Nacional de Desenvolvimento Científico e Tecnológico (CNPq; 304667/2016 $\square$, $791422441 / 2016 \square 3,303734 / 2012 \square 4,422410 / 2016 \square 0)$, Coordenação de Aperfeiçoamento

792 Pessoal de Nível Superior (CAPES, finance Code 001), Fundação de Amparo à

793 Pesquisa do Estado do Rio de Janeiro (FAPERJ, CNE 2015/E26/203.190/2015, PDR-

794 26/2002/010/2016), and PRPq-Universidade MG Federal MGPEFARJ, CNE

795 2015/E26/203.190/2015).

\section{Acknowledgments}

797 We would like to thank Alan Moraes for supporting the acquisition of electron

798 microscopy images and the electron microscopy laboratory at the UFF Biology Institute 
799 for allowing the use of the JEM1011 transmission electron microscope. We would also

800 like to thank Mauro Jorge Castro Cabral for the use of the MAGPIX® System

801 equipment at the Paulo de Góes Institute of Microbiology/UFRJ; Ernesto T. Marques Jr.

802 (Centro de Pesquisa Aggeu Magalhães, FIOCRUZ, PE) for providing ZIKV to the

803 Institute of Microbiology Paulo de Góes, Federal University of Rio de Janeiro; and the

804 technicians Juliana Gonçalves and Rakel Alves for their support during all the

805 experiments.

806 References

807

808 1. Garcez PP, Loiola EC, Madeiro da Costa R, Higa LM, Trindade P, Delvecchio R, et

809 al. Zika virus impairs growth in human neurospheres and brain organoids. Science.

810 2016;352(6287):816-8.

811 2. Brasil P, Calvet GA, Siqueira AM, Wakimoto M, de Sequeira PC, Nobre A, et al.

812 Zika Virus Outbreak in Rio de Janeiro, Brazil: Clinical Characterization, Epidemiological

813 and Virological Aspects. Powers AM, editor. PLoS Negl Trop Dis. 2016;10(4):e0004636.

814 3. Brasil P, Nielsen-Saines K. More pieces to the microcephaly-Zika virus puzzle in

815 Brazil. The Lancet Infectious Diseases. 2016;16(12):1307-9.

816 4. Brasil P, Pereira JP, Moreira ME, Ribeiro Nogueira RM, Damasceno L, Wakimoto

$817 \mathrm{M}$, et al. Zika Virus Infection in Pregnant Women in Rio de Janeiro. N Engl J Med. 2016

818 Mar 4;375(24):2321-34.

8195 5. Jaenisch T, Rosenberger KD, Brito C, Brady O, Brasil P, Marques ET. Risk of

820 microcephaly after Zika virus infection in Brazil, 2015 to 2016. Bull World Health

821 Organ. 2017;95(3):191-8.

822 6. Proenca-Modena JL, Milanez GP, Costa ML, Judice CC, Maranhão Costa FT. Zika

823 virus: lessons learned in Brazil. Microbes and Infection. 2018;20(11-12):661-9.

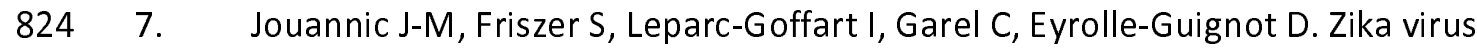

825 infection in French Polynesia. The Lancet. 2016;387(10023):1051-2.

826 8. Musso D, Ko Al, Baud D. Zika Virus Infection - After the Pandemic. Longo DL,

827 editor. N Engl J Med. 2019;381(15):1444-57.

828 9. Kasprzykowski Jl, Fukutani KF, Fabio H, Fukutani ER, Costa LC, Andrade BB, et al.

829 A recursive sub-typing screening surveillance system detects the appearance of the

830 ZIKV African lineage in Brazil: Is there a risk of a new epidemic? International Journal of

831 Infectious Diseases. 2020;96:579-81.

832 10. Miranda J, Martín-Tapia D, Valdespino-Vázquez Y, Alarcón L, Espejel-Nuñez A,

833 Guzmán-Huerta M, et al. Syncytiotrophoblast of Placentae from Women with Zika

834 Virus Infection Has Altered Tight Junction Protein Expression and Increased

835 Paracellular Permeability. Cells. 2019;8(10):1174.

836 11. Jurado KA, Simoni MK, Tang Z, Uraki R, Hwang J, Householder S, et al. Zika virus

837 productively infects primary human placenta-specific macrophages. JCI Insight.

838 2016;1(13):e88461. 
839 12. Quicke KM, Bowen JR, Johnson EL, McDonald CE, Ma H, O'Neal JT, et al. Zika 840 Virus Infects Human Placental Macrophages. Cell Host \& Microbe. 2016;20(1):83-90.

841 13. Tabata T, Petitt M, Puerta-Guardo H, Michlmayr D, Wang C, Fang-Hoover J, et

842 al. Zika Virus Targets Different Primary Human Placental Cells, Suggesting Two Routes

843 for Vertical Transmission. Cell Host \& Microbe. 2016;20(2):155-66.

844 14. Simoni MK, Jurado KA, Abrahams VM, Fikrig E, Guller S. Zika virus infection of

845 Hofbauer cells. Am J Reprod Immunol. 2017;77(2):e12613.

846 15. Rathore APS, Saron WAA, Lim T, Jahan N, John ALS. Maternal immunity and

847 antibodies to dengue virus promote infection and Zika virus-induced microcephaly in

848 fetuses. Science Advances. 2019; 5(2):eaav3208.

849 16. Lazear HM, Govero J, Smith AM, Platt DJ, Fernandez E, Miner JJ, et al. A Mouse

850 Model of Zika Virus Pathogenesis. Cell Host \& Microbe. 2016;19(5):720-30.

851 17. Grant A, Ponia SS, Tripathi S, Balasubramaniam V, Miorin L, Sourisseau M, et al.

852 Zika Virus Targets Human STAT2 to Inhibit Type I Interferon Signaling. Cell Host \&

853 Microbe. 2016;19(6):882-90.

854 18. Yockey L, Varela L, Rakib T, Khoury-Hanold W, Fink SL, Stutz B, et al. Vaginal

855 Exposure to Zika Virus during Pregnancy Leads to Fetal Brain Infection. Cell.

856 2016;166(5):1247-1256.e4.

857 19. Garcez PP, Stolp HB, Sravanam S, Christoff RR, Ferreira JCCG, Dias AA, et al. Zika

858 virus impairs the development of blood vessels in a mouse model of congenital

859 infection. Sci Rep. 2018;8(1):12774.

860 20. Elong Ngono A, Shresta S. Immune Response to Dengue and Zika. Annu Rev

861 Immunol. 2018;36(1):279-308.

862 21. Bayer A, Lennemann NJ, Ouyang Y, Bramley JC, Morosky S, Marques ETDAJ, et

863 al. Type III Interferons Produced by Human Placental Trophoblasts Confer Protection

864 against Zika Virus Infection. Cell Host Microbe. 2016;19(5):705-12.

865 22. Luo H, Winkelmann ER, Fernandez-Salas I, Li L, Mayer SV, Danis-Lozano R, et al.

866 Zika, dengue and yellow fever viruses induce differential anti-viral immune responses

867 in human monocytic and first trimester trophoblast cells. Antiviral Research.

868 2018;151:55-62.

869 23. Tanaka T, Narazaki M, Kishimoto T. IL-6 in Inflammation, Immunity, and

870 Disease. Cold Spring Harb Perspect Biol. 2014;6(10):a016295.

871 24. Hunter CA, Jones SA. IL-6 as a keystone cytokine in health and disease. Nature

872 Immunology. 2015;16(5):448-57.

873 25. Rabelo K, Gonçalves AJ da S, Souza U de, Sales AP, Lima SMB de, Trindade GF,

874 et al. Zika Virus Infects Human Placental Mast Cells and the HMC-1 Cell Line, and

875 Triggers Degranulation, Cytokine Release and Ultrastructural Changes. Cells.

$8762020 ; 9(4): 975-90$.

877 26. Bloise E, Ortiga-Carvalho TM, Reis FM, Lye SJ, Gibb W, Matthews SG. ATP-

878 binding cassette transporters in reproduction: a new frontier. Hum Reprod Update.

879 2015;22(2):164-81.

880 27. do Imperio GE, Bloise E, Javam M, Lye P, Constantinof A, Dunk C, et al.

881 Chorioamnionitis Induces a Specific Signature of Placental ABC Transporters Associated

882 with an Increase of miR-331-5p in the Human Preterm Placenta. Cell Physiol Biochem.

883 2018;45(2):591-604. 
884 28. Martinelli LM, Fontes KN, Reginatto MW, Andrade CBV, Monteiro VRS, Gomes

$885 \mathrm{HR}$, et al. Malaria in pregnancy regulates P-glycoprotein (P-gp/Abcb1a) and ABCA1

886 efflux transporters in the mouse visceral yolk sac. J Cell Mol Med. 2020;00:1-12.

887 29. Reginatto MW, Fontes KN, Monteiro VRS, Silva NL, Andrade CBV, Gomes HR, et

888 al. Effect of sub-lethal prenatal endotoxemia on murine placental transport systems

889 and lipid homeostasis. bioRxiv. 2020 Aug 5;2020.08.04.236745.

890 30. Martinelli LM, Reginatto MW, Fontes KN, Andrade CBV, Monteiro VRS, Gomes

$891 \mathrm{HR}$, et al. Breast cancer resistance protein (Bcrp/Abcg2) is selectively modulated by

892 lipopolysaccharide (LPS) in the mouse yolk sac. Reprod Toxicol. 2020; 98:82-91.

893 31. Imperio GE, Javam M, Lye P, Constantinof A, Dunk CE, Reis FM, et al.

894 Gestational age-dependent gene expression profiling of ATP-binding cassette

895 transporters in the healthy human placenta. J Cell Mol Med. 2019;23(1):610-8.

896 32. Vermillion MS, Lei J, Shabi Y, Baxter VK, Crilly NP, McLane M, et al. Intrauterine

897 Zika virus infection of pregnant immunocompetent mice models transplacental

898 transmission and adverse perinatal outcomes. Nat Commun. 2017;8(1):14575.

899 33. Szaba FM, Tighe M, Kummer LW, Lanzer KG, Ward JM, Lanthier P, et al. Zika

900 virus infection in immunocompetent pregnant mice causes fetal damage and placental

901 pathology in the absence of fetal infection. Coyne CB, editor. PLoS Pathog.

902 2018;14(4):e1006994.

903 34. Coelho SVA, Neris RLS, Papa MP, Schnellrath LC, Meuren LM, Tschoeke DA, et

904 al. Development of standard methods for Zika virus propagation, titration, and

905 purification. Journal of Virological Methods. 2017;246:65-74.

906 35. Connor KL, Kibschull M, Matysiak-Zablocki E, Nguyen TT-TN, Matthews SG, Lye

907 SJ, et al. Maternal malnutrition impacts placental morphology and transporter

908 expression: an origin for poor offspring growth. The Journal of Nutritional

909 Biochemistry. 2020;78:108329.

910 36. Fontes KN, Reginatto MW, Silva NL, Andrade CBV, Bloise FF, Monteiro VRS, et

911 al. Dysregulation of placental $A B C$ transporters in a murine model of malaria-induced

912 preterm labor. Sci Rep. 2019;9(1):11488.

913 37. Bloise E, Lin W, Liu X, Simbulan R, Kolahi KS, Petraglia F, et al. Impaired

914 Placental Nutrient Transport in Mice Generated by in Vitro Fertilization. Endocrinology.

915 2012;153(7):3457-67.

916 38. Lanciotti RS, Kosoy OL, Laven JJ, Velez JO, Lambert AJ, Johnson AJ, et al. Genetic

917 and Serologic Properties of Zika Virus Associated with an Epidemic, Yap State,

918 Micronesia, 2007. Emerg Infect Dis. 2008;14(8):1232-9.

919 39. Livak KJ, Schmittgen TD. Analysis of Relative Gene Expression Data Using Real-

920 Time Quantitative PCR and the 2- $\triangle \triangle C T$ Method. Methods. 2001;25(4):402-8.

921 40. Tschanz SA, Burri PH, Weibel ER. A simple tool for stereological assessment of

922 digital images: the STEPanizer: TOOL FOR STEREOLOGICAL ASSESSMENT. Journal of

923 Microscopy. 2011;243(1):47-59.

924 41. Sesso A, Belizário JE, Marques MM, Higuchi ML, Schumacher RI, Colquhoun A,

925 et al. Mitochondrial Swelling and Incipient Outer Membrane Rupture in Preapoptotic

926 and Apoptotic Cells. Anatomical Record. 2012;295(10):1647.

927 42. Montalbano R, Waldegger P, Quint K, Jabari S, Neureiter D, Illig R, et al.

928 Endoplasmic Reticulum Stress Plays a Pivotal Role in Cell Death Mediated by the Pan-

929 Deacetylase Inhibitor Panobinostat in Human Hepatocellular Cancer Cells.

930 Translational Oncology. 2013;6(2):143-IN6. 
931 43. Hamilton SA, Tower CL, Jones RL. Identification of Chemokines Associated with 932 the Recruitment of Decidual Leukocytes in Human Labour: Potential Novel Targets for 933 Preterm Labour. PLOS ONE. 2013;8(2):e56946.

934 44. Bloise E, Bhuiyan M, Audette MC, Petropoulos S, Javam M, Gibb W, et al.

935 Prenatal Endotoxemia and Placental Drug Transport in The Mouse: Placental Size-

936 Specific Effects. PLOS ONE. 2013;8(6):e65728.

937 45. Romero R, Mazor M, Brandt F, Sepulveda W, Avila C, Cotton DB, et al.

938 Interleukin-1 $\alpha$ and Interleukin-1 $\beta$ in Preterm and Term Human Parturition. American

939 Journal of Reproductive Immunology. 1992;27(3-4):117-23.

940 46. Larocca RA, Abbink P, Peron JPS, de A. Zanotto PM, Iampietro MJ, Badamchi-

941 Zadeh A, et al. Vaccine protection against Zika virus from Brazil. Nature.

942 2016;536(7617):474-8.

943 47. Souza IN de O, Frost PS, França JV, Nascimento-Viana JB, Neris RLS, Freitas L, et

944 al. Acute and chronic neurological consequences of early-life Zika virus infection in

945 mice. Science Translational Medicine. 2018;10(444):eaar2749.

946 48. Kalagiri RR, Carder T, Choudhury S, Vora N, Ballard AR, Govande V, et al.

947 Inflammation in Complicated Pregnancy and Its Outcome. Am J Perinatol.

948 2016;33(14):1337-56.

949 49. Barbeito-Andrés J, Pezzuto P, Higa LM, Dias AA, Vasconcelos JM, Santos TMP,

950 et al. Congenital Zika syndrome is associated with maternal protein malnutrition. Sci

951 Adv. 2020;6(2):eaaw6284.

952 50. Saito S, Nakashima A, Shima T, Ito M. REVIEW ARTICLE: Th1/Th2/Th17 and

953 Regulatory T-Cell Paradigm in Pregnancy. American Journal of Reproductive

954 Immunology. 2010;63(6):601-10.

955 51. Gotsch F, Romero R, Kusanovic JP, Mazaki-Tovi S, Pineles BL, Erez O, et al. The

956 fetal inflammatory response syndrome. Clin Obstet Gynecol. 2007;50(3):652-83.

957 52. Lucas CGO, Kitoko JZ, Ferreira FM, Suzart VG, Papa MP, Coelho SVA, et al.

958 Critical role of CD4 + T cells and IFN $\gamma$ signaling in antibody-mediated resistance to Zika

959 virus infection. Nature Communications. 2018;9(1):3136.

960 53. Burdet J, Sacerdoti F, Cella M, Franchi AM, Ibarra C. Role of TNF- $\alpha$ in the

961 mechanisms responsible for preterm delivery induced by Stx 2 in rats. British Journal of

962 Pharmacology. 2013;168(4):946.

963 54. Carpentier PA, Dingman AL, Palmer TD. Placental TNF- $\alpha$ Signaling in Illness-

964 Induced Complications of Pregnancy. The American Journal of Pathology.

965 2011;178(6):2802-10.

966 55. Romanowska-Próchnicka K, Felis-Giemza A, Olesińska M, Wojdasiewicz P,

967 Paradowska-Gorycka A, Szukiewicz D. The Role of TNF- $\alpha$ and Anti-TNF- $\alpha$ Agents during

968 Preconception, Pregnancy, and Breastfeeding. International Journal of Molecular

969 Sciences. 2021;22(6): 2922.

970 56. Sarr D, Bracken TC, Owino SO, Cooper CA, Smith GM, Nagy T, et al. Differential

971 roles of inflammation and apoptosis in initiation of mid-gestational abortion in

972 malaria-infected C57BL/6 and A/J mice. Placenta. 2015;36(7):738.

973 57. Galinsky R, Polglase GR, Hooper SB, Black MJ, Moss TJM. The consequences of

974 chorioamnionitis: preterm birth and effects on development. J Pregnancy.

975 2013;2013:412831.

976 58. Mor G, Cardenas I, Abrahams V, Guller S. Inflammation and pregnancy: the role

977 of the immune system at the implantation site. Ann N Y Acad Sci. 2011;1221(1):80-7. 
978 59. Ribeiro MR, Moreli JB, Marques RE, Papa MP, Meuren LM, Rahal P, et al. Zika979 virus-infected human full-term placental explants display pro-inflammatory responses 980 and undergo apoptosis. Arch Virol. 2018;163(10):2687-99.

981 60. Gown AM, Willingham MC. Improved Detection of Apoptotic Cells in Archival

982 Paraffin Sections: Immunohistochemistry Using Antibodies to Cleaved Caspase 3. J

983 Histochem Cytochem. 2002;50(4):449-54.

984 61. Noronha L de, Zanluca C, Burger M, Suzukawa AA, Azevedo M, Rebutini PZ, et

985 al. Zika Virus Infection at Different Pregnancy Stages: Anatomopathological Findings,

986 Target Cells and Viral Persistence in Placental Tissues. Front Microbiol.

987 2018;9(2266):1-11.

988 62. Lojpur T, Easton Z, Raez-Villanueva S, Laviolette S, Holloway AC, Hardy DB. $\Delta 9$ -

989 Tetrahydrocannabinol leads to endoplasmic reticulum stress and mitochondrial

990 dysfunction in human BeWo trophoblasts. Reproductive Toxicology. 2019;87:21-31.

991 63. Offerdahl DK, Dorward DW, Hansen BT, Bloom ME. Cytoarchitecture of Zika

992 virus infection in human neuroblastoma and Aedes albopictus cell lines. Virology.

993 2017;501:54-62.

994 64. Desuzinges-Mandon E, Arnaud O, Martinez L, Huché F, Di Pietro A, Falson P.

995 ABCG2 Transports and Transfers Heme to Albumin through Its Large Extracellular Loop.

996 J Biol Chem. 2010;285(43):33123-33.

997 65. Ledur PF, Karmirian K, Pedrosa C da SG, Souza LRQ, Assis-de-Lemos G, Martins

$998 \mathrm{TM}$, et al. Zika virus infection leads to mitochondrial failure, oxidative stress and DNA

999 damage in human iPSC-derived astrocytes. Scientific Reports. 2020;10(1):1218.

1000 66. Reemst K, Noctor SC, Lucassen PJ, Hol EM. The Indispensable Roles of Microglia

1001 and Astrocytes during Brain Development. Front Hum Neurosci. 2016;10:566.

1002 67. Guitart-Mampel M, Gonzalez-Tendero A, Niñerola S, Morén C, Catalán-Garcia

1003 M, González-Casacuberta I, et al. Cardiac and placental mitochondrial characterization

1004 in a rabbit model of intrauterine growth restriction. Biochimica et Biophysica Acta

1005 (BBA) - General Subjects. 2018;1862(5):1157-67.

1006 68. Guitart-Mampel M, Juarez-Flores DL, Youssef L, Moren C, Garcia-Otero L, Roca-

1007 Agujetas $\mathrm{V}$, et al. Mitochondrial implications in human pregnancies with intrauterine

1008 growth restriction and associated cardiac remodelling. J Cell Mol Med.

1009 2019;23(6):3962-73.

1010 69. Zhang Z, Rong L, Li Y-P. Flaviviridae Viruses and Oxidative Stress: Implications

1011 for Viral Pathogenesis. Oxid Med Cell Longev. 2019;2019:1409582.

1012 70. Martínez F, Kiriakidou M, Strauss JF. Structural and Functional Changes in

1013 Mitochondria Associated with Trophoblast Differentiation: Methods to Isolate

1014 Enriched Preparations of Syncytiotrophoblast Mitochondria. Endocrinology.

1015 1997;138(5):2172-83.

1016 71. Burton GJ, Yung HW, Murray AJ. Mitochondrial - Endoplasmic reticulum

1017 interactions in the trophoblast: Stress and senescence. Placenta. 2017;52:146-55.

1018 72. Holland O, Dekker Nitert M, Gallo LA, Vejzovic M, Fisher JJ, Perkins AV. Review:

1019 Placental mitochondrial function and structure in gestational disorders. Placenta.

1020 2017;54:2-9.

1021 73. Bridges CC, Zalups RK, Joshee L. Toxicological significance of renal Bcrp:

1022 Another potential transporter in the elimination of mercuric ions from proximal

1023 tubular cells. Toxicol Appl Pharmacol. 2015;285(2):110-7. 
1024 74. Szilagyi JT, Gorczyca L, Brinker A, Buckley B, Laskin JD, Aleksunes LM. Placental 1025 BCRP/ABCG2 Transporter Prevents Fetal Exposure to the Estrogenic Mycotoxin

1026 Zearalenone. Toxicol Sci. 2019;168(2):394-404.

1027 75. Scialis RJ, Aleksunes LM, Csanaky IL, Klaassen CD, Manautou JE. Identification 1028 and Characterization of Efflux Transporters That Modulate the Subtoxic Disposition of 1029 Diclofenac and Its Metabolites. Drug Metab Dispos. 2019;47(10):1080-92.

1030 76. Mao Q, Unadkat JD. Role of the breast cancer resistance protein (BCRP/ABCG2) 1031 in drug transport--an update. AAPS J. 2015;17(1):65-82.

1032 77. Lye P, Bloise E, Javam M, Gibb W, Lye SJ, Matthews SG. Impact of Bacterial and 1033 Viral Challenge on Multidrug Resistance in First- and Third-Trimester Human Placenta. 1034 The American Journal of Pathology. 2015;185(6):1666-75.

1035 78. Girard S, Sebire G. Transplacental Transfer of Interleukin-1 Receptor Agonist 1036 and Antagonist Following Maternal Immune Activation. American journal of 1037 reproductive immunology (New York, NY?: 1989). 2016;75(1):8-12.

1038 79. Bloise E, Petropoulos S, Iqbal M, Kostaki A, Ortiga-Carvalho TM, Gibb W, et al. 1039 Acute Effects of Viral Exposure on P-Glycoprotein Function in the Mouse Fetal Blood1040 Brain Barrier. Cellular physiology and biochemistry?: international journal of 1041 experimental cellular physiology, biochemistry, and pharmacology. 2017;41(3):1044104250. 